\title{
巯基类荧光探针的研究进展
}

\author{
闫沛沛*, $a$ 王 婷 $b$ 张 丹 ${ }^{b}$ 马晓雪 $a$ \\ $\left({ }^{a}\right.$ 山东医药技师学院 泰安 271000$)$ \\ ( ${ }^{b}$ 贵州大学化学与化工学院 贵阳 550000)
}

\begin{abstract}
摘要 生物硫醇类物质, 如半胱氨酸、高半胱氨酸、谷胱甘肽等, 在生命体中有至关重要的生理和病理作用. 因此, 近 几年对生物硫醇的研究成了一大热点. 目前颈基类苂光探针的设计主要是基于颈基的强亲核性. 根据苂光探针与颈基 反应的不同的机理，综述了近几年关于检测硫醇的苂光探针的合成报道，从迈克尔加成、醛基的环化、裂解、取代和 其他几个方面对探针的检测限、响应时间、当量比、选择性、稳定性等进行了综述.
\end{abstract}

关键词 統基; 苂光探针; 生物硫醇; 研究进展

\section{Progress in Fluorescence Sensors for Detection of Thiols}

\author{
Yan, Peipei ${ }^{*, a} \quad$ Wang, Ting ${ }^{b} \quad{\text { Zhang, } \text { Dan }^{b} \quad \text { Ma, Xiaoxue }}^{a}$ \\ ( ${ }^{a}$ Shandong Medical Technician College, Taian 271000) \\ ( ${ }^{b}$ School of Chemistry and Chemical Engineering, Guizhou University, Guiyang 550000)
}

\begin{abstract}
Sulfhydryl compounds, such as cysteine (Cys), homocysteine (Hcy), glutathione (GSH) and so on, play an important role in the normal human physiological processes. Molecular fluorescent probes have attracted much attention of scholar in the detection of sulfhydryl compounds, since it has the advantage of good selectivity, high sensitivity, good biocompatibility and real-time in situ monitoring. At present, the design of thiol-based fluorescent probes is mainly based on the strong nucleophilicity of sulfhydryl groups. In this paper, the synthesis of fluorescent probes for detecting sulfhydryl compounds, which was reported from 2013 to 2018, is reviewed based on the different mechanism of the reaction between fluorescence probe and sulfhydryl, including Michael addition, cyclization of aldehyde groups, sulfonamides or sulfonates of the lysis, natural chemical linkage and so on. The detection limit of the probe, response time, equivalence ratio were elaborated.
\end{abstract}

Keywords thiols; fluorescent probe; biological thiols; research progress

生物硫醇, 如半胱氨酸 $(\mathrm{Cys})$ 、高半胱氨酸(Hcy)、谷 胱甘肽(GSH), 在各个生理过程中起着至关重要的作用, 因此对生命体中的生物硫醇进行定量的跟踪分析监测 成为近几年来的研究热点 ${ }^{[1 \sim 3]}$. 半胱氨酸是生命体中非 常常见的一种氨基酸, 存在多种蛋白质和多肽中; 具有 淡化黑色素、延缓衰老、促进体制生长等作用 ${ }^{[4,5]}$. 半胱 氨酸缺乏与许多疾病密切相关, 如儿童生长缓慢 ${ }^{[6]}$ 、毛

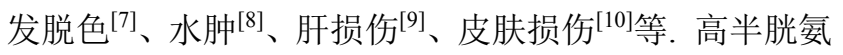
酸又称同型半胱氨酸, 是半胱氨酸的异种, 高水平的同 型半胱氨酸称为高同型半胱氨酸血症, 会导致人体的认 知功能障碍 ${ }^{[1]}$, 严重者会导致阿尔茨海默病和精神分裂

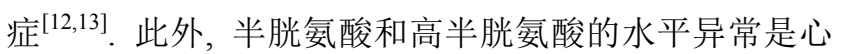
血管疾病的重要原因之一 ${ }^{[14,15]}$. 谷胱甘肽几乎存在于人
体每一个细胞中, 它具有维持正常免疫功能、调整氧化 作用、整合解毒作用等功能 ${ }^{[16,17]}$. 因此对生命体中生物 硫醇的分析检测具有非常重要的生理和病理意义.

目前, 硫醇检测的主要分析技术包括高效液相色谱

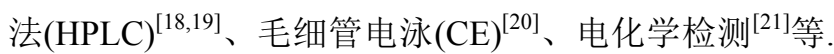
而这些分析技术成本高，选择性差，精度低. 因此，苂 光探针法因其简单、低成本、高选择性和适用于细胞内 硫醇的检测而被广泛报道.

目前, 用于硫醇检测的荧光探针的设计原理主要是 基于其强亲核性和对金属离子的高亲和性. 本文综述了 迈克尔加成反应、醛类环化、裂解、取代等反应的机理, 探讨了硫醇类化合物探针的最新研究进展.

\footnotetext{
* Corresponding author. E-mail: 1458851831@qq.com

Received July 31, 2018; revised October 11, 2018; published online December 28, 2018.
} 


\section{1 基于迈克尔加成反应}

1978 年, Sippel ${ }^{[22]}$ 首次报道了, 用迈克尔加成的反 应机理设计合成了用于检测大鼠体内生物硫醇的荧光 探针. 此后该方法成为了设计合成硫醇类苂光探针的热 门 ${ }^{[23,24]}$, 并经过近几年的研究在选择性、稳定性、灵敏 度等方面取得了突破性进展. 虽然现在生物硫醇总量的 检测取得了突破性进展, 但是在单一硫醇的选择性监 测、探针的灵敏度等方面仍然是个艰巨的挑战 ${ }^{[25]}$.

2017 年, Fan 等 ${ }^{[26]}$ 设计报道的光诱导电子转移 (Photo induced Electron Transfer, PET)体系的苂光探针 $\mathbf{1}$ (Scheme 1). 当探针 1 在与颈基类物质识别之前, 由于 PET 效应探针苂光基团的苂光大幅减弱; 当探针与颈基 类物质通过迈克尔加成反应识别之后, 荧光探针的 PET 过程被阻断, 进而使得探针荧光基团的荧光恢复. 在 $\mathrm{CH}_{3} \mathrm{CN} / \mathrm{H}_{2} \mathrm{O}(V: V=1: 1,20 \mathrm{mmol} / \mathrm{L}$ PBS, $\mathrm{pH}$ 7.4) 的溶 液中, 探针 1 在在波长为 $525 \mathrm{~nm}$ 处发出较强的苂光(激 发波长为 $360 \mathrm{~nm}$ ). 加入 Cys 后 $525 \mathrm{~nm}$ 处的荧光发射急 剧减少, 当加入的 Cys 达到探针 $\mathbf{1}$ 的 50 equiv. 时, 荧光 几乎被完全萃灭, 与探针 $\mathbf{1}$ 单独存在时相比荧光值下降 了近 20 equiv. 探针 $\mathbf{1}$ 与 Cys 响应后 $2 \mathrm{~h}$ 荧光值就会被完 全萃灭, 而探针 1 与 $\mathrm{Hcy}$ 的响应时间大约为 $3 \mathrm{~h}$, 与 $\mathrm{GSH}$ 的响应时间则更长. 因此可以利用探针对三中颈基化合 物响应时间的不同来区分鉴别三种氨基酸. 此外探针 $\mathbf{1}$ 已经成功地被应用于 A375 细胞中 Cys 的检测.

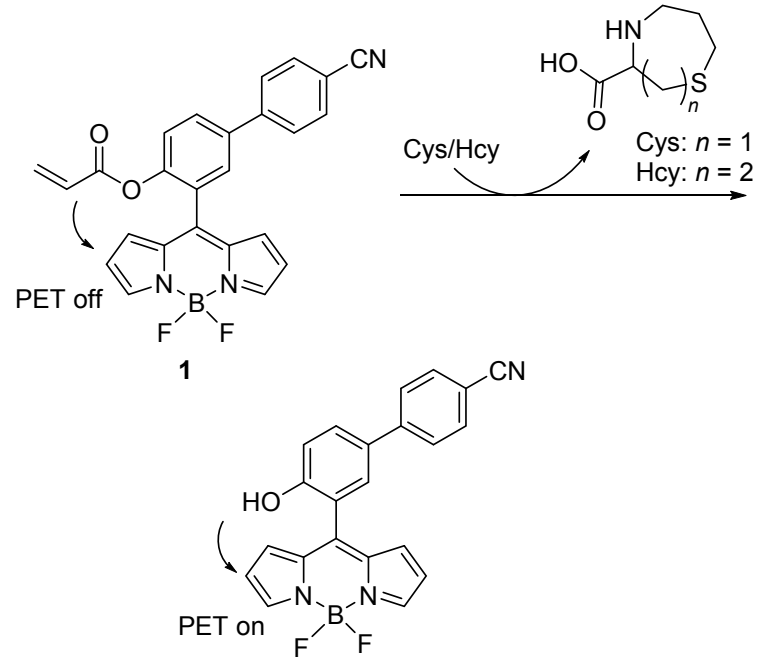

图式 1 探针 1 与生物硫醇识别示意图

Scheme 1 Schematic illustration of reaction of 1 with thiols

2014 年, Dai 等 ${ }^{[27]}$ 报道了一种检测 Cys 的基于香豆 素和吡唑啉的比例型探针 2 (图 2). 相较于其他的氨基 酸, 包括谷胱甘肽和同型半胱氨酸, 探针 $\mathbf{2}$ 对半胱氨酸 有很高的选择性. 在探针 $\mathbf{2}\left(10 \mu \mathrm{mol} \cdot \mathrm{L}^{-1}\right)$ 磷酸缓冲溶液 $\left(\mathrm{CH}_{3} \mathrm{CN}: \mathrm{PBS}=1: 1, \mathrm{pH} 7.4\right)$ 中, 加入不同浓度的 Cys
$(0 \sim 13$ equiv.), 随着 Cys 浓度的增加, 荧光强度在 560 $\mathrm{nm}$ 处逐渐增强, 直至苂光值增加 9 equiv., 且检测限为 $5.08 \mu \mathrm{mol} \cdot \mathrm{L}^{-1}$. 探针 2 对 Cys 的响应时间为 $40 \mathrm{~min}$. 另 外, 探针 2 已经被成功应用于 A549 细胞中 Cys 的检测.

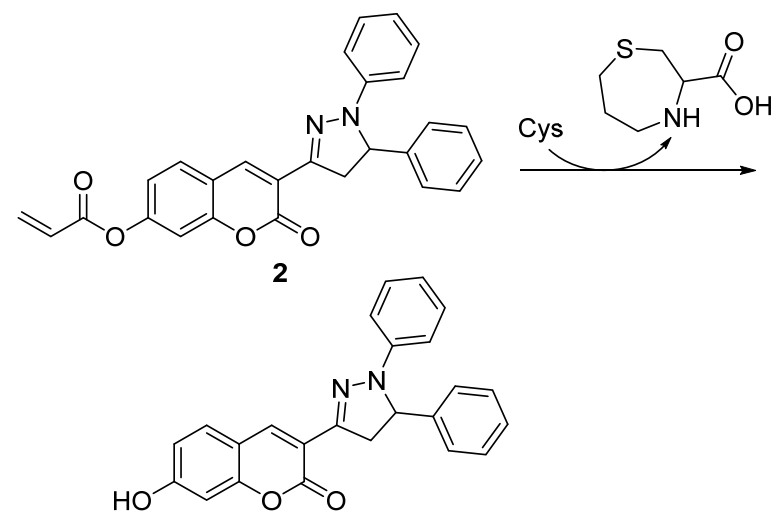

图 2 探针 2 与半胱氨酸识别示意图

Figure 2 Schematic illustration of reaction of $\mathbf{2}$ with Cys

2013 年, Lim 等 ${ }^{[28]}$ 报道了检测 Cys 的罗丹明类荧光 探针 3 (图 3). 探针 3 表现出对 Cys 的高选择性，可以有 效从各种氨基酸(包括 GSH 和 Hcy)中鉴别出 Cys. 探针 3 与 $\mathrm{Cys}$ 的响应速率较快 $\left(k^{2}=0.0176 \mathrm{~mol} \cdot \mathrm{L}^{-1} \cdot \mathrm{s}^{-1}\right)$, 说明 探针 3 的灵敏度较高. 在探针 $3\left(20 \mu \mathrm{mol} \cdot \mathrm{L}^{-1}\right)$ 的 HEPES 缓冲溶液 $\left(0.10 \mathrm{~mol} \cdot \mathrm{L}^{-1}, \mathrm{pH} 7.4\right)$ 中, 加入不同浓度的 Cys $\left(0 \sim 20 \mathrm{mmol} \cdot \mathrm{L}^{-1}\right)$, 在波长为 $525 \mathrm{~nm}$ 处的苂光值随着 Cys 浓度的增加而增加, 最终达到峰值, 检测限低至 $7.62 \mu \mathrm{mol} \cdot \mathrm{L}^{-1}$. 另外细胞成像实验表明，探针 3 能够对 活细胞中的 Cys 进行成像检测.<smiles>C=C1[C+]=CC(=C)C(C(=O)OC(=O)C=CC)=C1</smiles>

图 3 探针 3 与半胱氨酸识别示意图

Figure 3 Schematic illustration of reaction of $\mathbf{3}$ with Cys

2014 年, Huo 等 ${ }^{[29]}$ 研究报道了一种检测硫脲类化合 物的荧光探针 4 (图 4). 探针 4 与 GSH 和 Cys 识别后发 出较强的绿色荧光, 而与 Hcy 识别后的荧光强度较弱, 与其他氨基酸几乎不响应. 在探针 $4\left(10 \mu \mathrm{mol} \cdot \mathrm{L}^{-1}\right)$ 的 $\mathrm{DMSO} / \mathrm{H}_{2} \mathrm{O}\left(V: V=1: 3,10 \mathrm{mmol} \cdot \mathrm{L}^{-1} \mathrm{HEPES}, \mathrm{pH}\right.$ 7.4 $)$ 溶液中, 加入不同浓度的 $\mathrm{GSH}\left(0 \sim 60 \mu \mathrm{mol} \cdot \mathrm{L}^{-1}\right)$. 实验 
结果表明，探针 4 与 GSH 识别后的苂光强度与 GSH 的 浓度呈线性关系, 检测限为 $0.026 \mu \mathrm{mol} \cdot \mathrm{L}^{-1}$. 此外, 探针 4 已经成功应用于活细胞中颈基的标记.

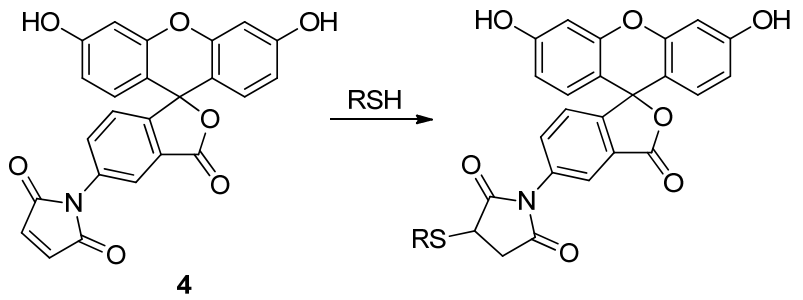

图 4 探针 4 与硫醇类物质识别示意图

Figure 4 Schematic illustration of reaction of 4 with thiols

2014 年, $\mathrm{Qu}$ 等 ${ }^{\left[{ }^{30]}\right.}$ 报道了检测生物硫醇的马来酰亚 胺类的苂光探针 5 (图 5). 探针 $\mathbf{5}$ 对生物硫醇类物质表现 为较高的选择性. 探针 $\mathbf{5}\left(0.6 \mu \mathrm{mol} \cdot \mathrm{L}^{-1}\right)$ 与生物硫醇 $(10$ $\left.\mu \mathrm{mol} \cdot \mathrm{L}^{-1}\right)$ 的响应时间监测实验表明，探针 5 与 Cys、 Hcy、GSH 的反应均在 $20 \mathrm{~s}$ 内完成, 说明探针 5 对生物 硫醇的检测具有很高的灵敏度. 在探针 $\mathbf{5}\left(0.6 \mu \mathrm{mol} \cdot \mathrm{L}^{-1}\right)$ 的 HEPES $(10 \mathrm{mmol} / \mathrm{L}, \mathrm{pH}$ 7.4)缓冲溶液中加入不同浓度 的 Hcy $\left(0 \sim 0.6 \mu \mathrm{mol} \cdot \mathrm{L}^{-1}\right)$, 随着 Hcy 浓度的增加荧光强 度不断增加直至达到峰值, 检测限在 $10^{-8} \sim 10^{-7} \mathrm{~mol} \cdot$ $\mathrm{L}^{-1}$ 之间. 此外, 探针 5 具有良好的细胞膜穿透性, 可用 于标记活细胞中的 Cys、Hcy 和 GSH.
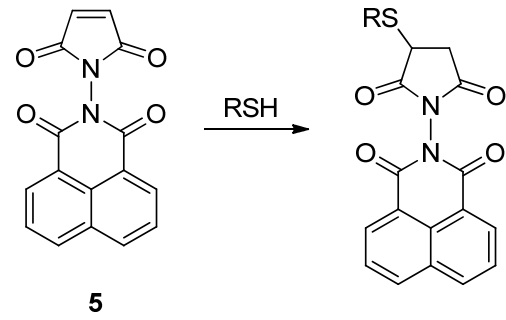

图 5 探针 5 与生物硫醇类物质识别示意图

Figure 5 Schematic illustration of reaction of 5 with bio-thiols

2015 年, Shen 等 ${ }^{[31]}$ 报道了一种含 7-硝基-2-氧代 -1,3-二唑和 4-马来酰亚胺苯酚片段的用于检测生物硫 醇的苂光探针 6 (图 6). 探针 6 对生物硫醇类物质具有较 好的选择性. 探针 $6\left(5 \mu \mathrm{mol} \cdot \mathrm{L}^{-1}\right)$ 的 $\mathrm{CH}_{3} \mathrm{CN} / \mathrm{H}_{2} \mathrm{O}(V$ : $\left.V=1: 9,50 \mathrm{mmol} \cdot \mathrm{L}^{-1} \mathrm{PBS}, \mathrm{pH} 7.0\right)$ 溶液几乎无荧光发 射, 当加入 Cys $\left(70 \mu \mathrm{mol} \cdot \mathrm{L}^{-1}\right)$ 后随着时间的推移其苂光 强度不断增加, $15 \mathrm{~min}$ 后达到峰值. 探针 $\mathbf{6}$ 与不同浓度 的 Cys 响应实验表明, 探针 6 可以定量检测 Cys, 检测 限为 $12 \times 10^{-7} \mathrm{~mol} \cdot \mathrm{L}^{-1}$. 此外, 探针 6 已经成功地用于对 $\mathrm{HeLa}$ 细胞中硫醇的成像.

2016 年, Liu 等 ${ }^{[32]}$ 报道了一种制备简便的检测生物 硫醇的荧光探针 7 (图 7). 探针 7 本身是没有荧光发射现 象的, 当加入 $\mathrm{GSH}$ 后在 $480 \mathrm{~nm}$ 处出现一个很强的苂光

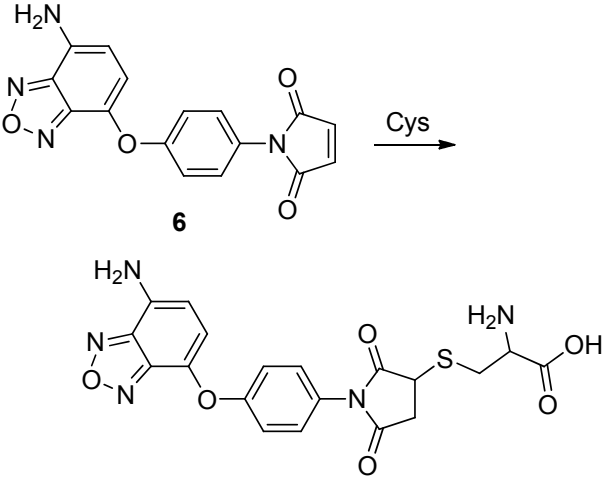

图 6 探针 6 与半胱氨酸类物质识别示意图

Figure 6 Schematic illustration of reaction of $\mathbf{6}$ with Cys

发射峰，荧光值相较于没有加入 GSH 之前增加了近 24 equiv. 探针 7 与 GSH 和 Hcy 的响应时间均在 $75 \mathrm{~s}$ 之内, 与 Cys 的响应时间为 $150 \mathrm{~s}$. 在探针 $7\left(0.5 \mu \mathrm{mol} \cdot \mathrm{L}^{-1}\right)$ 的 $\mathrm{CH}_{3} \mathrm{OH} / \mathrm{H}_{2} \mathrm{O}(V: V=1: 1,10 \mathrm{mmol} / \mathrm{L}$ HEPES, $\mathrm{pH}$ 7.4)中 加入 GSH $\left(0 \sim 3.5 \mu \mathrm{mol} \cdot \mathrm{L}^{-1}\right)$, 其苂光值与 GSH 的浓度 程较好的线性关系，检测限为 $0.085 \mathrm{mmol} / \mathrm{L}$. 此外，探 针 7 已经成功应用于 HepG2 细胞中生物硫醇的检测.

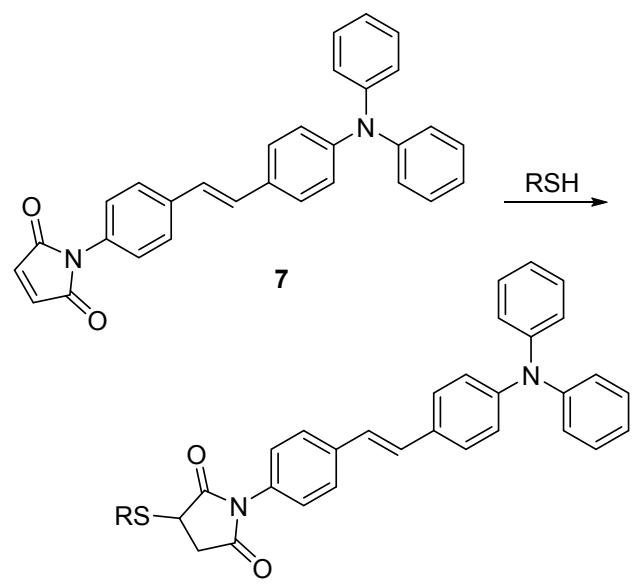

图 7 探针 7 与生物硫醇类物质识别示意图

Figure 7 Schematic illustration of reaction of 7 with thiols

2016 年, Zhao 等 ${ }^{[33]}$ 报道了用于检测生物硫醇类物 质的苂光探针 8 (图 8). 探针 8 的 ESIPT 苂光团被连接的 马来酰亚胺触发 PET 效应，使得探针本身无苂光发射现 象，而当統基与马来酰亚胺发生迈克尔加成反应后， PET 效应被阻断进而使得 ESIPT 荧光团的荧光恢复, 并 伴有较大的 Stokes 位移. 探针 8 的溶液中加入 Cys, $30 \mathrm{~s}$ 内苂光值就能达到 Cys 之前的荧光值的 23 equiv.的峰 值. 在探针 $8\left(2 \mu \mathrm{mol} \cdot \mathrm{L}^{-1}\right)$ 的 $\mathrm{PBS}$ 缓冲溶液 $(10 \mathrm{mmol} \cdot$ $\left.\mathrm{L}^{-1}, \mathrm{pH} 7.4,0.1 \% \mathrm{DMSO}\right)$ 中加入 Cys $\left(0 \sim 3.2 \mu \mathrm{mol} \cdot \mathrm{L}^{-1}\right)$, 随着 Cys 浓度的增加苂光强度逐渐增加, 检测限为 $3.78 \times 10^{-8} \mathrm{~mol} \cdot \mathrm{L}^{-1}$. 此外, 探针 $\mathbf{8}$ 成功的应用于活细胞 中生物硫醇的检测. 


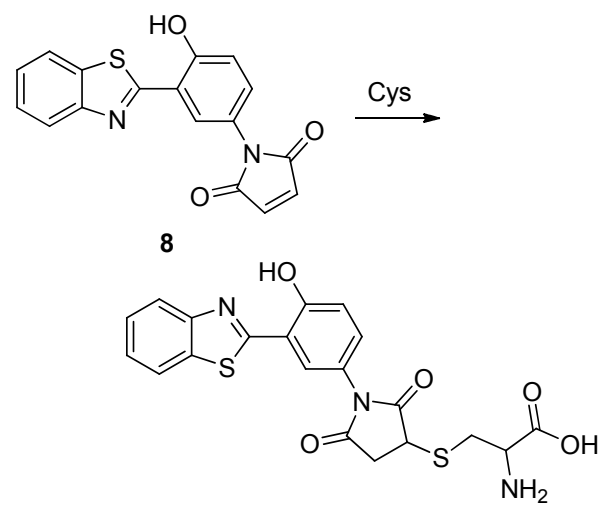

图 8 探针 8 与半胱氨酸识别示意图

Figure 8 Schematic illustration of reaction of $\mathbf{8}$ with Cys.

2017 年, Yue 等 ${ }^{[34]}$ 报道了一种可以同时检测 Cys 及 其代谢产物 $\mathrm{SO}_{2}$ 的荧光探针 9 (图 9). 探针 9 对 $\mathrm{Cys}$ 表现 出极高的选择性, 甚至可以从结构较为相似的 Hcy 和 $\mathrm{GSH}$ 中区分检测出 Cys. 探针 $9\left(10 \mu \mathrm{mol} \cdot \mathrm{L}^{-1}\right)$ 和 Cys $(20$ $\mu \mathrm{mol} \cdot \mathrm{L}^{-1}$ )在 $100 \mathrm{~s}$ 内苂光值达到峰值, 在发射峰为 514 $\mathrm{nm}$ 处苂光值最大增量为 130 equiv. 此外, 探针 9 与 Cys 响应的苂光值和 Cys 的浓度呈较好的线性关系, 检测限 为 $0.46 \mu \mathrm{mol} \cdot \mathrm{L}^{-1}$. 更重要的是, 探针 9 可以用于观察在 A549 细胞中 Cys 向 $\mathrm{SO}_{2}$ 的转化, 并且已成功地应用于斑 马鱼的外源性半胱氨酸和 $\mathrm{SO}_{2}$ 成像.<smiles></smiles>

图 9 探针 9 与半胱氨酸和 $\mathrm{SO}_{2}$ 识别示意图

Figure 9 Schematic illustration of reaction of 9 with Cys and $\mathrm{SO}_{2}$

2017 年, Sok 等 ${ }^{[35]}$ 报道了两种检测 GSH 的具有 $\alpha, \beta-$ 不饱和羰基的高效荧光探针 10 和 11 (图 10). 探针 10 和
11 对 GSH 都有较好的选择性. 其中探针 11 (5 $\mu \mathrm{mol} ・$ $\left.\mathrm{L}^{-1}\right)$ 的发射苂光随 $\mathrm{GSH}$ 浓度 $\left(0 \sim 5 \mu \mathrm{mol} \cdot \mathrm{L}^{-1}\right)$ 呈非线性 增加, 这意味着探针可能有助于评价硫醇含量. 将 GSH $\left(1 \mu \mathrm{mol} \cdot \mathrm{L}^{-1}\right)$ 分别加入到探针 $10\left(5 \mu \mathrm{mol} \cdot \mathrm{L}^{-1}\right)$ 和探针 11 $\left(5 \mu \mathrm{mol} \cdot \mathrm{L}^{-1}\right)$ 的 $\mathrm{PBS}$ 缓冲溶液 $\left(0.1 \mathrm{mmol} \cdot \mathrm{L}^{-1}, \mathrm{pH}\right.$ 7.4)中, 监测 $320 \mathrm{~nm}$ 处的吸光度变化, 探针 $\mathbf{1 1}$ 中的 GSH 在 20 $\min$ 被消耗完全, 而探针 $\mathbf{1 0}$ 中的 GSH 在 $130 \mathrm{~min}$ 后被 消耗了 $20 \%$. 总体来看, 探针 10 和 11 都可以较好地检 测 GSH，其中探针 11 的检测性能更好.

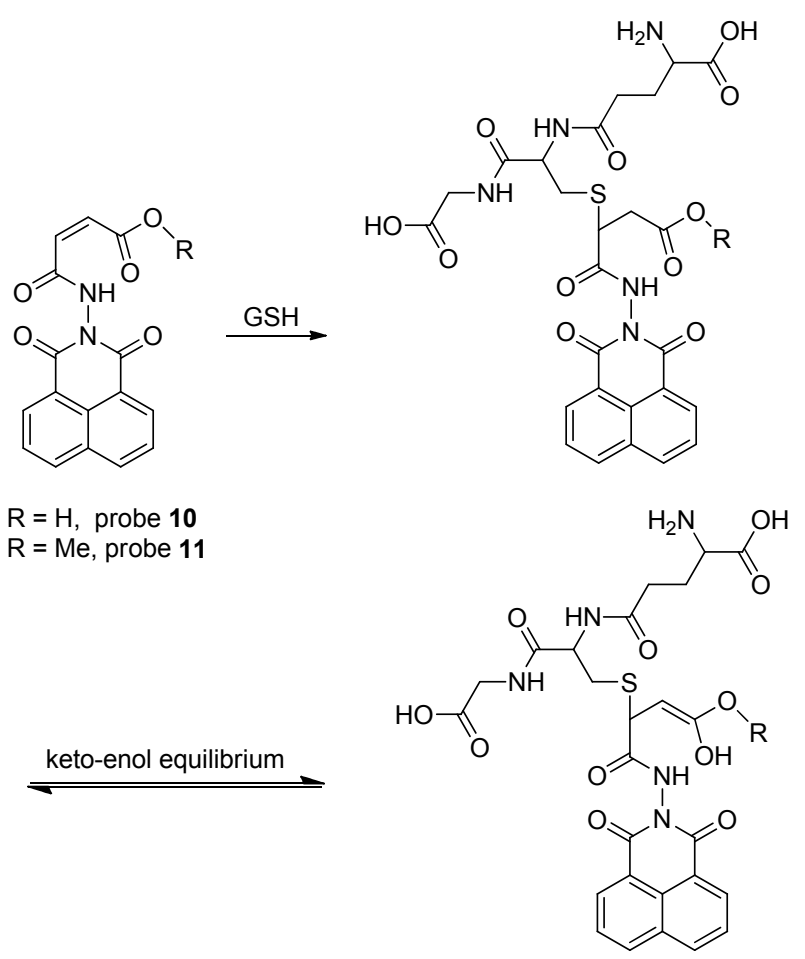

图 10 探针 10 和探针 11 与谷胱甘肽识别示意图 Figure 10 Schematic illustration of reaction of $\mathbf{1 0}$ and $\mathbf{1 1}$ with GSH.<smiles>[R]SC(c1c(O)ccc2cc(C(=O)OCC)c(=O)oc12)C(C(C)=O)C(C)=O</smiles>

图 11 探针 12 与生物硫醇类物质识别示意图

Figure 11 Schematic illustration of reaction of $\mathbf{1 2}$ with thiols

2013 年, Long 等 ${ }^{[36]}$ 报道了检测生物硫醇的基于迈 克尔加成的香豆素类苂光探针 12(图 11). 在探针 12 (5 $\left.\mu \mathrm{mol} \cdot \mathrm{L}^{-1}\right)$ 的磷酸钾缓冲溶液 $\left(10 \mathrm{mmol} \cdot \mathrm{L}^{-1}, \mathrm{pH} 7.4,2 \%\right.$ DMSO)中加入不同浓度的 Cys $\left(0 \sim 350 \mu \mathrm{mol} \cdot \mathrm{L}^{-1}\right)$, 其在 发射波长为 $455 \mathrm{~nm}$ 处的荧光值随 $\mathrm{Cys}$ 的浓度增加而增 加, 检测限为 $0.22 \mu \mathrm{mol} \cdot \mathrm{L}^{-1} .5 \mu \mathrm{mol} \cdot \mathrm{L}^{-1}$ 的探针 12 与 
$350 \mu \mathrm{mol} \cdot \mathrm{L}^{-1}$ 的 Cys、Hcy、GSH 的响应时间分别为 1.5 、 5 和 $10 \mathrm{~min}$. 此外, 探针 $\mathbf{1 2}$ 已经被成功应用于人血清中 生物硫醇的检测.

2015 年, Dai 等 ${ }^{\left[{ }^{37]}\right.}$ 报道了一系列基于颈基与不饱和 酮的迈克尔加成反应的苂光探针 $13 \sim 22$. 探针 $13 \sim 17$ 是 7 号位被取代的 2-(喹啉-2-亚甲基)丙二酸类物质, 而 探针 18 22 是 7 号位被取代的 2-(喹啉-2-亚甲基)丙二 酸乙酯类物质(图 12). 两类探针的 $\mathrm{pH}$ 适应性不同，探针 $13 \sim 17$ 可以在 $\mathrm{pH}<7$ 的条件下检测硫醇类物质，探针 $18 \sim 22$ 可以在 $\mathrm{pH}>7$ 的条件下检测硫醇类物质. 其中, 在探针 $13\left(20 \mu \mathrm{mol} \cdot \mathrm{L}^{-1}\right)$ 的 $\mathrm{DMSO} / 0.1 \mathrm{~mol} \cdot \mathrm{L}^{-1}$ 的磷酸柠 檬酸缓冲溶液 $(V: V=2: 98, \mathrm{pH}$ 5) 加入 Cys $(80 \mu \mathrm{mol} \cdot$ $\left.\mathrm{L}^{-1}\right), 20 \mathrm{~min}$ 内苂光值达到原来的 96 equiv. 的峰值. 此 外, 由于探针 13 和 14 的高度敏感和选择性可以检测活 细胞中的硫醇物质, 并且这两个探针已经成功标记活细 胞中的溶酶体.
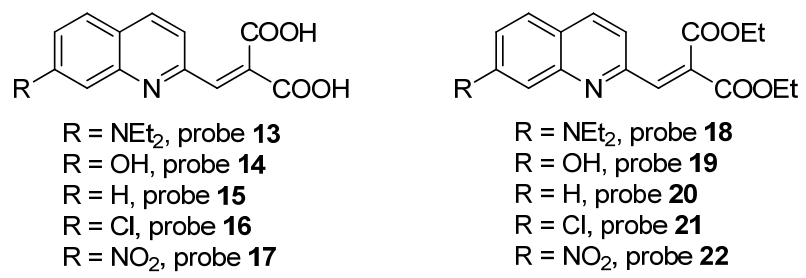

图 12 探针 13 22 的结构示意图

Figure 12 Chemical Structures of Two Kinds of Probes, 13 22

\section{2 基于醛的环化}

2016 年, Yang 等 ${ }^{[38]}$ 报道了一种检测 GSH 的高水溶 性的苂光探针 23(图 13). 该探针通过引入羧基来提高探 针的水溶性, 减少了探针用于活细胞检测时有机溶剂对 细胞的损伤. 探针 23 对 GSH 具有较高的选择性, 不受 其它两种生物颈基(Cys、Hcy)和其他 19 种氨基酸的干 扰. 探针 $23\left(10 \mu \mathrm{mol} \cdot \mathrm{L}^{-1}\right)$ 与 $\mathrm{GSH}\left(25 \mu \mathrm{mol} \cdot \mathrm{L}^{-1}\right)$ 响应 90 $\mathrm{s}$ 内荧光值增加近 70 equiv., 说明探针与 GSH 响应的速 率快且具有较大的当量比. 在探针 $23\left(10 \mu \mathrm{mol} \cdot \mathrm{L}^{-1}\right)$ 的 PBS 缓冲溶液中加入不同浓度的 GSH $(0 \sim 25 \mu \mathrm{mol} \cdot$ $\mathrm{L}^{-1}$ ), 其荧光值与 $\mathrm{GSH}$ 的浓度程线性关系. 探针 $\mathbf{2 3}$ 为提 高罗丹明类荧光探针的水溶性提供了新的方法. 另外, 探针 23 已经被成功应用于活细胞中 GSH 的检测.

2014 年, Meng 等 ${ }^{[39]}$ 报道了一种检测 Cys 和 Hcy 的 双光子的苂光探针 24 (图 14). 探针 $\mathbf{2 4}$ 的荧光发射波长 在 $505 \mathrm{~nm}$, 当加入 Cys 后由于 ICT 效应荧光发射波长蓝 移至 $410 \mathrm{~nm}$. 探针对 Cys 和 Hcy 有较好的选择性, 与其 他巯基类物质均无响应. 在探针 $24\left(25 \mu \mathrm{mol} \cdot \mathrm{L}^{-1}\right)$ 的 PBS/DMSO $(V: V=1: 9, \mathrm{pH}$ 7.4) 溶液中加入不同浓度 的 Cys $\left(0 \sim 80 \mu \mathrm{mol} \cdot \mathrm{L}^{-1}\right)$, 在波长为 $410 \mathrm{~nm}$ 处荧光值随

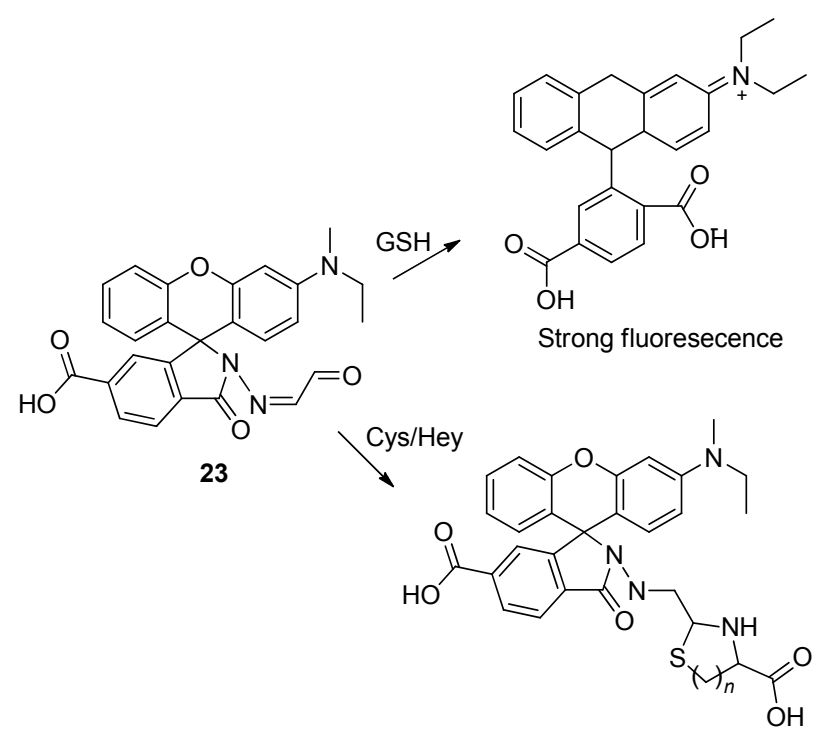

图 13 探针 23 与谷胱甘肽识别示意图

Figure 13 Schematic illustration of reaction of $\mathbf{2 3}$ with thiols

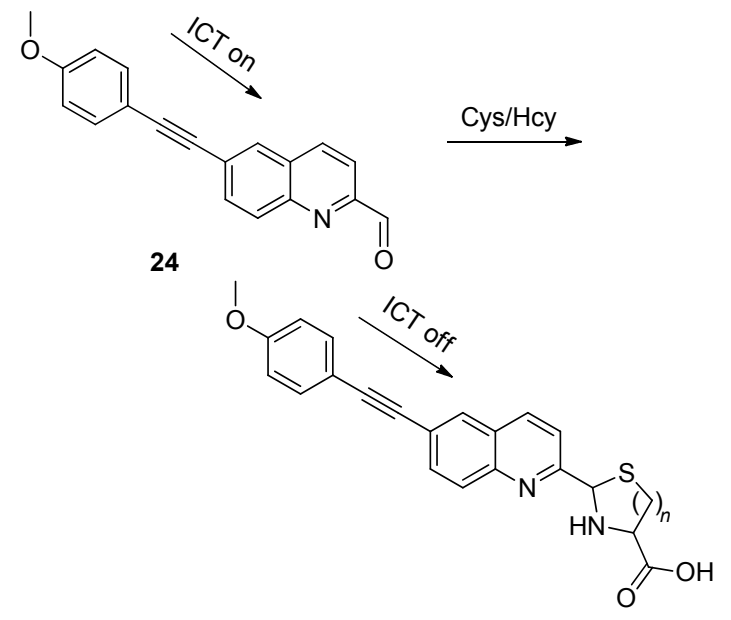

图 14 探针 24 与 $\mathrm{Cys} / \mathrm{Hcy}$ 识别示意图

Figure 14 Schematic illustration of reaction of 24 with Cys/ Hcy

着 Cys 浓度的增加而增加, 而在波长为 $505 \mathrm{~nm}$ 处荧光 值随 Cys 浓度的增加而降低. 探针 $24\left(10 \mu \mathrm{mol} \cdot \mathrm{L}^{-1}\right)$ 和 Cys $\left(100 \mu \mathrm{mol} \cdot \mathrm{L}^{-1}\right)$ 反应约 $6 \mathrm{~min}$ 结束. 此外，探针 $\mathbf{2 4}$ 提 供了一种简单且可见的检测 Cys 的方法，并能实时地检 测双光子激发.

2016 年, Chen 等 ${ }^{\left[{ }^{[0]}\right.}$ 报道了一种检测生物硫醇的香 豆素类荧光探针 $\mathbf{2 5}$ (图 15). 探针 $\mathbf{2 5}$ 只对 Cys 和 Hcy 有 荧光响应，对包括 GSH 在内的其他氨基酸几乎无荧光 响应. 室温下探针 $25\left(10 \mu \mathrm{mol} \cdot \mathrm{L}^{-1}\right)$ 与 Cys (20 equiv.)混 合 $10 \mathrm{~min}$ 后，苂光值有明显的增加, $30 \mathrm{~min}$ 后荧光值基 本达到峰值. 在探针 $25\left(10 \mu \mathrm{mol} \cdot \mathrm{L}^{-1}\right)$ 的 $\mathrm{PBS}$ 缓冲溶液 $\left(20 \mathrm{mmol} \cdot \mathrm{L}^{-1}, \mathrm{pH}\right.$ 7.4) 中加入不同浓度的 Cys $(0 \sim 70$ equiv.), 荧光值随着 Cys 浓度的增加而增大, 检测限为 
$0.874 \mu \mathrm{mol} \cdot \mathrm{L}^{-1}$. 此外, 探针 $\mathbf{2 5}$ 已经成功地用于 $\mathrm{HeLa}$ 细 胞中生物硫醇的苂光成像.

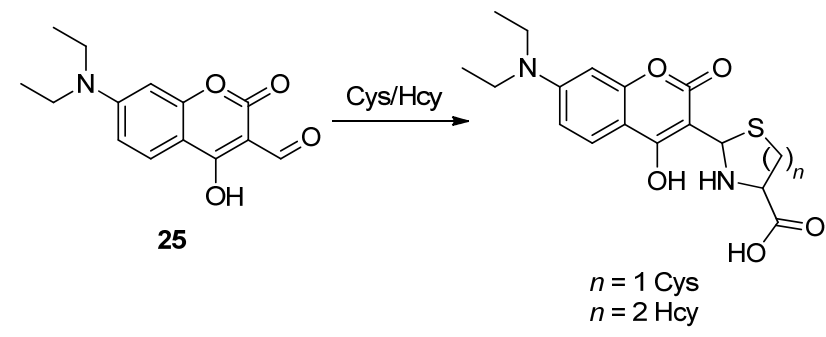

图 15 探针 $\mathbf{2 5}$ 与 $\mathrm{Cys} / \mathrm{Hcy}$ 识别示意图

Figure 15 Schematic illustration of reaction of $\mathbf{2 5}$ with Cys/ Hcy

2016 年, Zhang 等 ${ }^{[41]}$ 报道了基于 PET 效应的荧光探 针 26 (图 16). 探针 26 对 Cys 和 Hcy 的检测选择性很高. 在探针 26 的 DMSO/PBS 缓冲溶液 $(V: V=7: 3,10$ $\mathrm{mmol} \cdot \mathrm{L}^{-1}, \mathrm{pH}$ 7.4)中加入不同浓度的, 探针的苂光值均 随 Cys 或 Hcy 浓度的增加而增加，检测限分别为 0.82 和 $1.45 \mu \mathrm{mol} \cdot \mathrm{L}^{-1}$. 探针 $26\left(10 \mu \mathrm{mol} \cdot \mathrm{L}^{-1}\right)$ 分别于 Cys $(3$ $\left.\mathrm{mmol} \cdot \mathrm{L}^{-1}\right)$ 和 Hcy $\left(5 \mathrm{mmol} \cdot \mathrm{L}^{-1}\right)$ 反应, 荧光值分别在 30 和 $40 \mathrm{~min}$ 后达到峰值. 此外, 初步的生物荧光成像结果 表明，探针 26 具有良好的生物相容性.<smiles></smiles>

图 16 探针 26 与 $\mathrm{Cys} / \mathrm{Hcy}$ 识别示意图

Figure 16 Schematic illustration of reaction of $\mathbf{2 6}$ with Cys/ Hcy

2013 年, Kong 等 ${ }^{[42]}$ 报道了一种检测 $\mathrm{Cys} / \mathrm{Hcy}$ 的高选 择性的近红外苂光探针 27(图 17). 近红外荧光探针的发 射波长在 $600 \sim 1000 \mathrm{~nm}$ 之间, 这类荧光探针有效地避 开了生物细胞中自身荧光的干扰. 在探针 $27(2 \mu \mathrm{mol}$ $\mathrm{L}^{-1}$ ) 的 PBS 缓冲溶液 $\left(40 \mathrm{mmol} \cdot \mathrm{L}^{-1}, \mathrm{PH}\right.$ 7.4)中加入不同 浓度的 Cys $\left(0 \sim 2 \mu \mathrm{mol} \cdot \mathrm{L}^{-1}\right)$, 其荧光值在波长为 $778 \mathrm{~nm}$ 处随着 Cys 浓度的增加而增加, 检测限低至 $7.9 \times 10^{-9}$ $\mathrm{mol} \cdot \mathrm{L}^{-1}$. 此外, 探针 27 已经被成功地用于在活的 HepG2 细胞中成像内源性 Cys/Hcy.

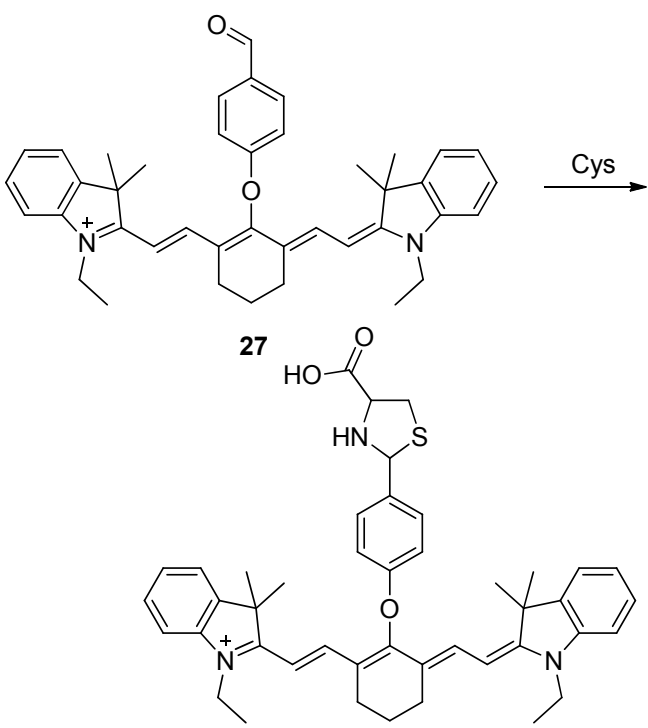

图 17 探针 27 与半胱氨酸识别示意图

Figure 17 Schematic illustration of reaction of $\mathbf{2 7}$ with Cys

2016 年, Gong 等 ${ }^{[43]}$ 报道了一种基于 ICT 机制的苂 光探针 28(图 18). 探针 28 对 Cys 具有较好的选择性, 包 括 Hcy 和 GSH 在内的其他氨基酸与探针 $\mathbf{2 8}$ 几乎不反应. 在探针 $28\left(20 \mu \mathrm{mol} \cdot \mathrm{L}^{-1}\right)$ 的 $\mathrm{CH}_{3} \mathrm{CN} / \mathrm{PBS}$ 缓冲溶液 $(V$ : $\left.V=2: 8,10 \mathrm{mmol} \cdot \mathrm{L}^{-1}, \mathrm{pH} 7.4\right)$ 中加入不同浓度的 Cys $\left(0 \sim 700 \mu \mathrm{mol} \cdot \mathrm{L}^{-1}\right)$, 探针 28 的苂光强度会随着 $\mathrm{Cys}$ 的增 加而增加, 检测限为 $54.6 \mathrm{nmol} \cdot \mathrm{L}^{-1}$. 探针 28 与 50 equiv. Cys 反应 $5 \mathrm{~min}$ 即可达到饱和, 说明探针具有很高的灵 敏度. 此外, 探针 $\mathbf{2 8}$ 已经成功地应用于活细胞内的 Cys 的生物成像.

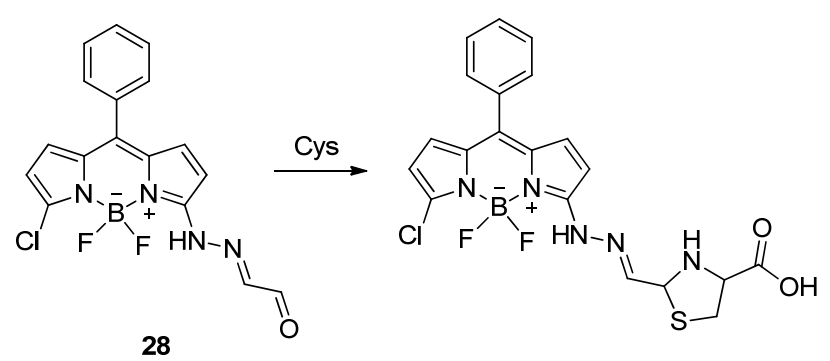

图 18 探针 28 与半胱氨酸识别示意图

Figure 18 Schematic illustration of reaction of $\mathbf{2 8}$ with Cys

2017 年, Gao 等 ${ }^{[4]}$ 报道了含醛基的金属铱复合物苂 光探针 29(图 19). 探针 29 对 Hcy 具有较好的选择性, 可 以有效地区分 Hcy 与其他两种生物硫醇(Cys 和 GSH). 探针 $29\left(10 \mu \mathrm{mol} \cdot \mathrm{L}^{-1}\right)$ 的荧光值与 Hcy 的浓度 $(0 \sim 700$ $\left.\mu \mathrm{mol} \cdot \mathrm{L}^{-1}\right)$ 成正比, 检测限为 $4.6 \mu \mathrm{mol} \cdot \mathrm{L}^{-1}$. 此外, 探针 29 具有明显的细胞通透性和低细胞毒性, 并且已经成 功地应用于活细胞中 Hcy 的成像. 探针 29 的优良性能, 使其在对细胞中 Hcy 的跟踪研究方面具有巨大的潜力. 


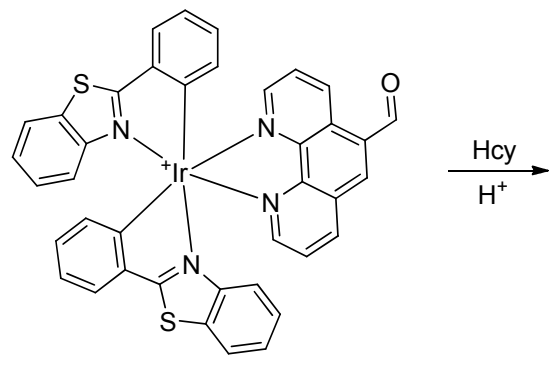

29

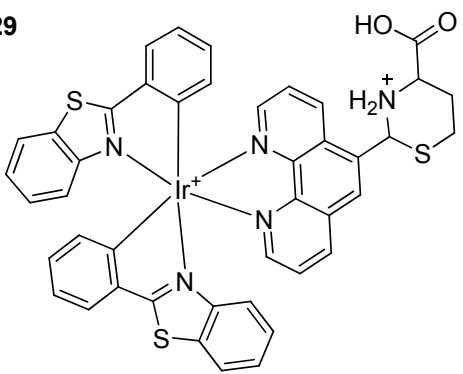

图 19 探针 29 与半胱氨酸识别示意图

Figure 19 Schematic illustration of reaction of $\mathbf{2 9}$ with Hcy

2017 年, Kim 等 ${ }^{[45]}$ 报道了一种检测 Cys 的可回收的 荧光探针 30 (图 20). 探针 30 与 Cys 在水溶液中发生环 化反应, 同时伴随着颜色由橙色变为黄色. 然而当探针 30 与 Cys 反应后的产物与采离子识别, 又会产生探针 30, 因此探针 30 可以重复利用. 探针 30 对 Cys 的选择 性非常好, 甚至包括结构较为相似的 Hcy 和 GSH 在内 的其他氨基酸对探针识别 Cys 均无干扰. 探针 $\mathbf{3 0}$ 对 Cys 的检测的良好性能, 说明探针 $\mathbf{3 0}$ 具有应用于细胞内 Cys 检测的潜力.

\section{3 基于裂解反应}

2016 年, Wang 等 ${ }^{[46]}$ 报道了一种检测生物硫醇的 PET 效应的荧光关-开探针 31 (图 21). 探针 31 对生物硫 醇具有较好的选择性, 基本不受其他几种氨基酸的干 扰. 当 $\mathrm{pH}=3.5$ 时, 探针 30 只对 $\mathrm{Cys}$ 有响应, 而当 $\mathrm{pH}=5.5$ 时, 探针 31 对 Cys、Hcy、GSH 均有响应. 在 探针 $31\left(10 \mu \mathrm{mol} \cdot \mathrm{L}^{-1}\right)$ 的 $\mathrm{DMSO} / \mathrm{H}_{2} \mathrm{O}$ 溶液 $(V: V=9: 1$, $\left.10 \mathrm{mmol} \cdot \mathrm{L}^{-1} \mathrm{PBS}, \mathrm{pH}=3.5\right)$ 中加入不同浓度的 Cys $(0 \sim$ 15 equiv.), 探针 31 的苂光值与 Cys 的浓度呈线性关系, 检测限为 $0.117 \mu \mathrm{mol} \cdot \mathrm{L}^{-1}$. 在 $\mathrm{pH}=3.5$ 的条件下, 探针 $31\left(10 \mu \mathrm{mol} \cdot \mathrm{L}^{-1}\right)$ 与 Cys (15 equiv.) 反应 $80 \mathrm{~min}$ 后达到饱 和. 此外, 探针 31 已成功检测到 HeLa 细胞中的硫醇.

2017 年, Chen 等 ${ }^{[47]}$ 报道了一种具有较大 Stokes 位 移(202 nm) 的荧光探针 32 (图 22). 探针 32 由于 PET 和 ESIPT 效应基本无苂光发射, 当加入 Cys 后, 探针的 PET 效应被阻断在 $482 \mathrm{~nm}$ 处的苂光值显著增加. 在探 针 $32\left(10 \mu \mathrm{mol} \cdot \mathrm{L}^{-1}\right)$ 的 $\mathrm{HEPES} / \mathrm{DMSO}$ 溶液 $(V: V=4: 1$, $\left.50.0 \mathrm{mmol} \bullet \mathrm{L}^{-1}, \mathrm{pH} 7.4\right)$ 中加入不同浓度的 Cys $(0 \sim 80$

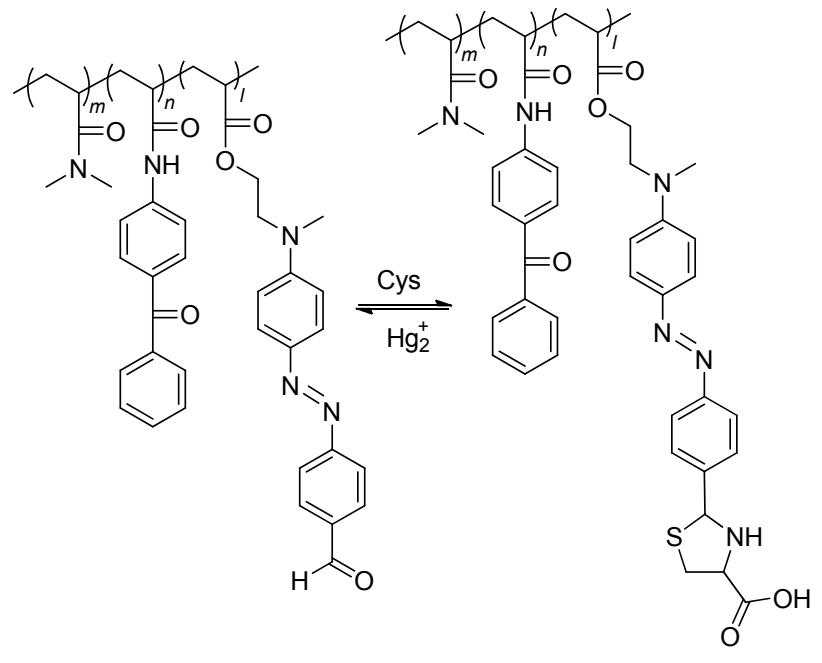

图 20 探针 30 与半胱氨酸识别示意图

Figure 20 Schematic illustration of reaction of $\mathbf{3 0}$ with Cys

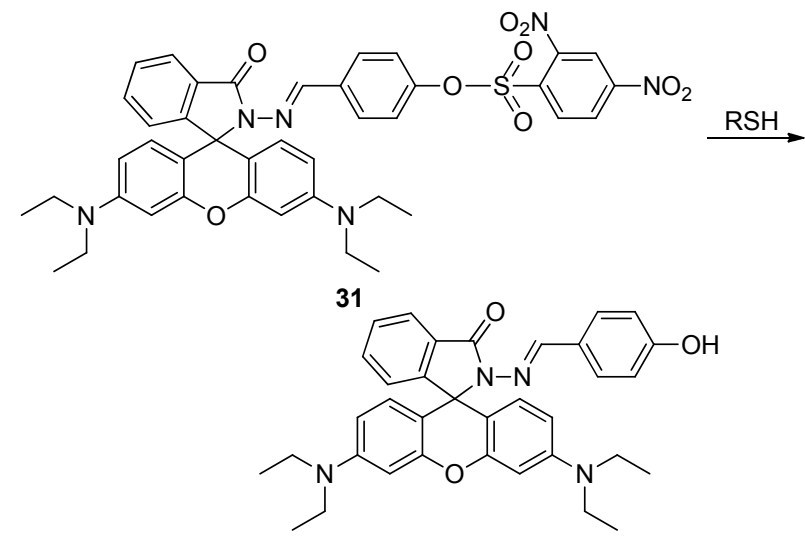

图 21 探针 $\mathbf{3 1}$ 与生物硫醇类物质识别示意图

Figure 21 Schematic illustration of reaction of $\mathbf{3 1}$ with biothiols

$\mu \mathrm{mol} \cdot \mathrm{L}^{-1}$ ), 探针的苂光值会随着 Cys 浓度增加而增加, 检测限为 $2 \times 10^{-8} \mathrm{~mol} \cdot \mathrm{L}^{-1}$. 探针 $32\left(10 \mu \mathrm{mol} \cdot \mathrm{L}^{-1}\right)$ 与 $\mathrm{Cys}$ $\left(80 \mu \mathrm{mol} \cdot \mathrm{L}^{-1}\right)$ 反应 $30 \mathrm{~min}$ 内苂光值增加近 20 equiv. 峰 值. 此外, 探针 32 已经被成功应用于 NCI-H226 细胞中 生物硫醇的检测.

2013 年, Wei 等 ${ }^{[48]}$ 报道了一种检测 Cys 和 GSH 的高 选择性的苂光探针 33 (图 23). 探针 33 对生物硫醇的检 测具有较好的选择性和稳定性, 响应的程度大小为 $\mathrm{GSH}>\mathrm{Cys}>\mathrm{Hcy}$. 在探针 33 的磷酸盐缓冲溶液 $(10$ $\left.\mu \mathrm{mol} \cdot \mathrm{L}^{-1}\right)$ 中分别加入不同浓度的 Cys $\left(0 \sim 60 \mu \mathrm{mol} \bullet \mathrm{L}^{-1}\right)$ 和 GSH $\left(0 \sim 60 \mu \mathrm{mol} \cdot \mathrm{L}^{-1}\right)$, 探针 33 的荧光强度与 $\mathrm{Cys}$ 和 GSH 的浓度程线性关系, 线性相关系数分别为 0.9956 和 0.9966. 此外, 根据探针 33 在细胞中检测生物硫醇的 结果判断，其在疾病诊断中具有潜在的应用前景.

2016 年, Liu 等 ${ }^{[49]}$ 报道了检测生物硫醇的高选择性 苂光探针 34 (图 24). 探针 34 的溶液中加入生物硫醇后 


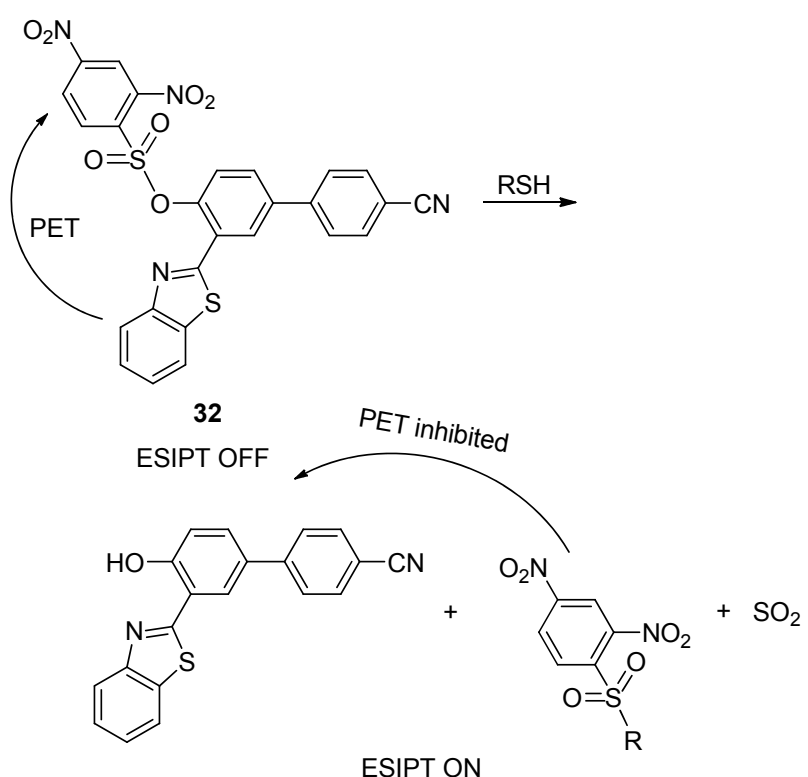

图 22 探针 32 与生物硫醇类物质识别示意图

Figure 22 Schematic illustration of reaction of $\mathbf{3 2}$ with biothiols

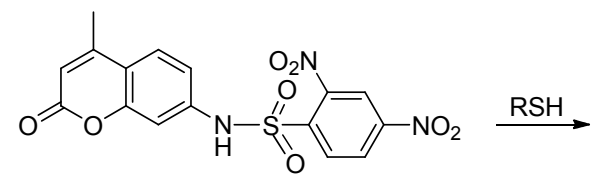

33<smiles>Cc1cc(=O)oc2cc(N)ccc12</smiles>

图 23 探针 33 与生物硫醇类物质识别示意图

Figure 23 Schematic illustration of reaction of $\mathbf{3 3}$ with thiols<smiles>[R]S(=O)(=O)c1ccc([N+](=O)[O-])cc1[N+](=O)[O-]</smiles>

图 24 探针 $\mathbf{3 4}$ 与生物硫醇类物质识别示意图

Figure 24 Schematic illustration of reaction of $\mathbf{3 4}$ with biothiols

肉眼可见其颜色由无色变为黄色, 其苂光从无苂光变为 强黄色苂光. 在探针 $34\left(10 \mu \mathrm{mol} \cdot \mathrm{L}^{-1}\right)$ 的 PBS 缓冲溶液 $\left(10 \mathrm{mmol} \cdot \mathrm{L}^{-1}, \mathrm{pH}=7.4\right)$ 中加入 $\mathrm{Cys}\left(0 \sim 3 \mu \mathrm{mol} \cdot \mathrm{L}^{-1}\right)$, 其 荧光值会随 Cys 浓度增加而增加, 检测限为 $0.16 \mu \mathrm{mol}$ -
$\mathrm{L}^{-1}$. 探针 $34\left(10 \mu \mathrm{mol} \cdot \mathrm{L}^{-1}\right)$ 和生物硫醇 (5 equiv.) 反应 1 $\min$ 后苂光强度急剧增加, 反应 $5 \mathrm{~min}$ 后苂光值达到饱 和. 所有数据表明探针 34 在生物成像中显示出潜在用 途应用.

2016 年, Fan 等 ${ }^{[50]}$ 报道了一种检测生物硫醇的双光 子苂光探针 35(图 25). 双光子荧光探针因为其光毒性 小、穿透力强、可视性强等优点一直是生物荧光探针领 域的研究重点. 探针 35 与 Cys 的响应时间约为 $20 \mathrm{~min}$, 而与 GSH 和 Hcy 的响应时间相对要长一点, 且探针 35 与 Cys 相应的最大苂光值增加了近 74 equiv. 在探针 $\mathbf{3 5}$ $\left(10 \mu \mathrm{mol} \cdot \mathrm{L}^{-1}\right)$ 的 DMSO/HEPES $(V: V=3: 7,10$ $\mathrm{mmol} \bullet \mathrm{L}^{-1}, \mathrm{pH}$ 7.4)溶液中加入不同浓度的 Cys $(0 \sim 500$ $\mu \mathrm{mol} \cdot \mathrm{L}^{-1}$ ), 探针 35 的苂光强度与 $\mathrm{Cys}$ 的浓度程线性关 系, 检测限为 $0.26 \mu \mathrm{mol} \cdot \mathrm{L}^{-1}$. 此外, 探针 35 已经成功地 应用于活细胞中生物硫醇的检测.
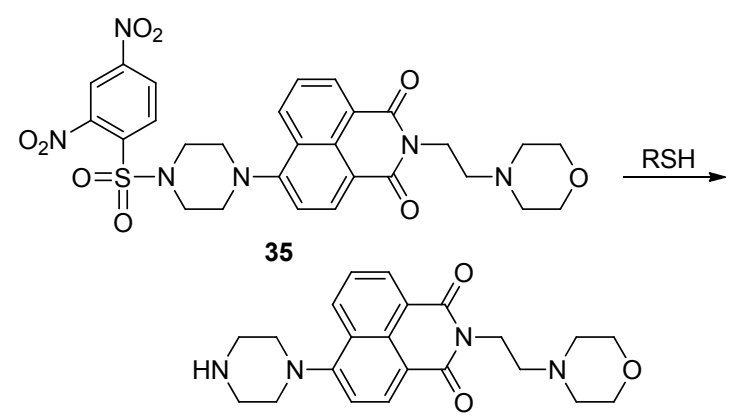

图 25 探针 35 与生物硫醇类物质识别示意图

Figure 25 Schematic illustration of reaction of $\mathbf{3 5}$ with biothiols

2014 年, Yoshida 等 ${ }^{[51]}$ 报道了一种检测生物硫醇的 高灵敏度的荧光探针 36(图 26), 该探针可以在短时间内 监测细胞中生物硫醇的水平. 探针 $36\left(1 \mu \mathrm{mol} \cdot \mathrm{L}^{-1}\right)$ 与 GSH $\left(10 \mu \mathrm{mol} \cdot \mathrm{L}^{-1}\right)$ 响应 $1 \mathrm{~h}$ 后, 荧光值及达到饱和, 比 没有加入 GSH 之前的苂光值增加了近 6000 equiv.之多. 更重要的是, 该探针已经成功地检测到小鼠卵巢的癌细 胞中的生物硫醇.

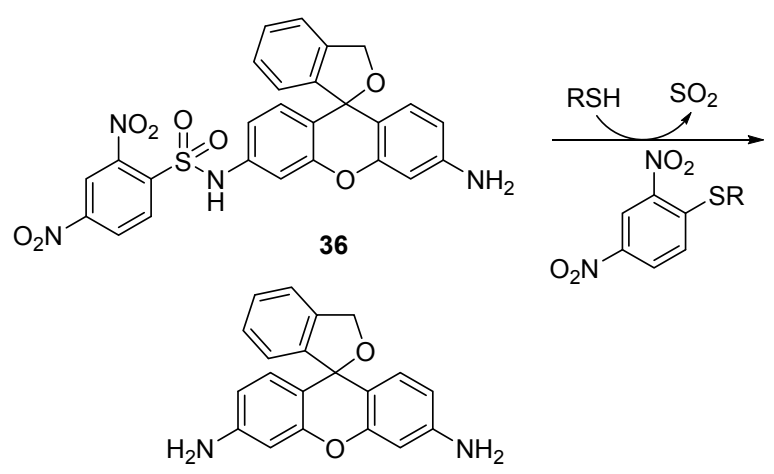

图 26 探针 36 与生物硫醇类物质识别示意图

Figure 26 Schematic illustration of reaction of $\mathbf{3 6}$ with biothiols 
2017 年, Wang 等 ${ }^{[52]}$ 报道了一种监测生物硫醇的近 红外菼光探针 37 (图 27). 将 GSH $\left(0 \sim 25 \mu \mathrm{mol} \cdot \mathrm{L}^{-1}\right)$ 滴定 到探针 $37\left(10 \mu \mathrm{mol} \cdot \mathrm{L}^{-1}\right)$ 的 PBS $\left(10 \mathrm{mmol} \cdot \mathrm{L}^{-1}, \mathrm{pH} 7.4\right.$, $50 \% \mathrm{DMSO}$ )缓冲溶液中, 在 $676 \mathrm{~nm}$ 处可观察到苂光强 度逐渐增强, 检测限低至 $1.19 \mathrm{nmol} \cdot \mathrm{L}^{-1}$. 此外探针 37 与 GSH 的响应非常迅速, 将 GSH $\left(2 \mu \mathrm{mol} \cdot \mathrm{L}^{-1}\right)$ 加入到探针 $37\left(10 \mu \mathrm{mol} \cdot \mathrm{L}^{-1}\right)$ 的溶液中, $6 \mathrm{~min}$ 荧光值即可达到峰值. 更重要的是, 探针 37 已经成功应用于活细胞中 GSH 的 检测.<smiles>CC1(C)CC(/C=C/c2ccc(O)cc2)=CC(=C(C#N)C#N)CC1(C)C</smiles><smiles>CC(C)(C)OC(C)(C)C</smiles>

Strong fluorescence

图 27 探针 $\mathbf{3 7}$ 与生物硫醇类物质识别示意图

Figure 27 Schematic illustration of reaction of $\mathbf{3 7}$ with biothiols

2013 年, $\mathrm{Xu}$ 等 ${ }^{[53]}$ 报道了一种可循环利用的检测 GSH 的近红外苂光探针 38 (图 28). 在探针 38 (10 $\mu \mathrm{mol}$ $\left.\mathrm{L}^{-1}\right)$ 的 HEPES (10 $\mathrm{mmol} \cdot \mathrm{L}^{-1}, \mathrm{pH}$ 7.4)溶液中加入不同浓 度的 GSH $\left(0 \sim 20 \mu \mathrm{mol} \cdot \mathrm{L}^{-1}\right)$, 探针 38 的苂光值在 794 $\mathrm{nm}$ 处随 GSH 浓度的增加而减弱, 而当在探针 $\mathbf{3 8}$ 与 $\mathrm{GSH}$ $\left(20 \mu \mathrm{mol} \cdot \mathrm{L}^{-1}\right)$ 的反应产物中加入不同浓度的 $\mathrm{H}_{2} \mathrm{O}_{2}(0 \sim$ $200 \mu \mathrm{mol} \cdot \mathrm{L}^{-1}$ ) 时, 其荧光值在 $794 \mathrm{~nm}$ 处又会随 $\mathrm{H}_{2} \mathrm{O}_{2}$ 浓 度的增加而增加. 当 GSH $\left(20 \mu \mathrm{mol} \cdot \mathrm{L}^{-1}\right)$ 加入到探针 38

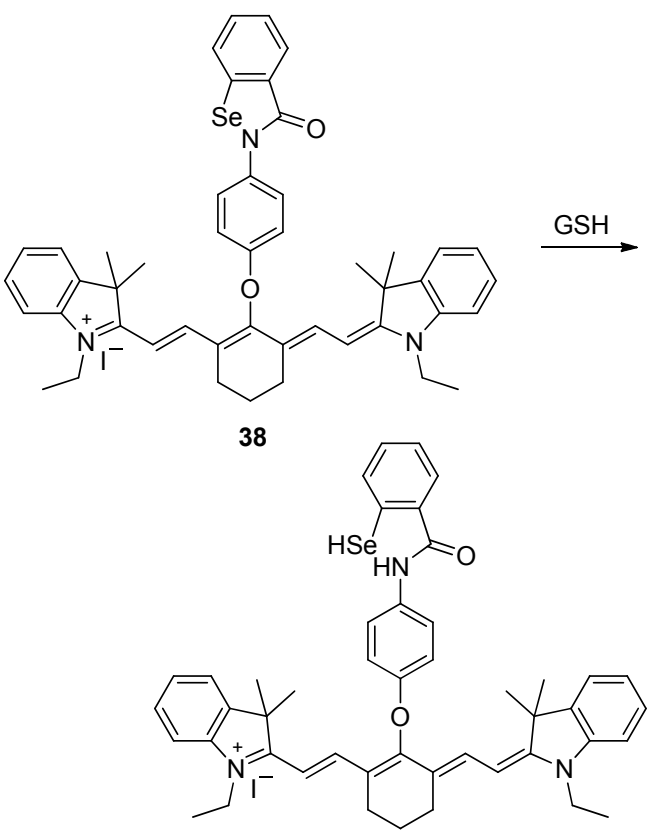

图 28 探针 38 与谷胱甘肽识别示意图

Figure 28 Schematic illustration of reaction of $\mathbf{3 8}$ with GSH

$\left(10 \mu \mathrm{mol} \cdot \mathrm{L}^{-1}\right)$ 的溶液中, $5 \mathrm{~min}$ 后荧光值发生明显的减 小. 此外, 探针 38 实现了细胞调亡过程中 GSH 的氧化 还原过程的实时监测.

\section{4 基于取代反应}

2014 年, Zheng 等 ${ }^{[54]}$ 报道了检测生物硫醇的 FRET 效应的荧光探针 39 (图 29). 苂光探针 39 的苂光由于 FRET 效应被大大减弱, 当探针 39 与 Cys 响应后 FRET 效应被阻断菼光恢复. 在探针 $39\left(10 \mu \mathrm{mol} \cdot \mathrm{L}^{-1}\right)$ 的溶液 中加入不同浓度的 Cys $\left(0 \sim 40 \mu \mathrm{mol} \cdot \mathrm{L}^{-1}\right)$, 苂光值随 Cys 浓度的增加而增加，检测限为 $0.8 \mu \mathrm{mol} \cdot \mathrm{L}^{-1}$. 探针 39 (10 $\left.\mu \mathrm{mol} \cdot \mathrm{L}^{-1}\right)$ 与 Cys $\left(10 \mu \mathrm{mol} \cdot \mathrm{L}^{-1}\right)$ 响应 $16 \mathrm{~min}$ 后苂光值达 到饱和. 此外, 探针 39 已经成功地应用于复方氨基酸注 射液中 Cys 浓度的测定.

2013 年, $\operatorname{Lim}$ 等 $^{[55]}$ 报道了一种检测生物硫醇的荧光 探针 40 (图 30). 探针 40 对生物硫醇表现出良好的选择 性和灵敏度, 并且探针 40 具有较好的水溶性. 在探针<smiles>[R]OSCCCNCC(=S)Nc1ccc2c(c1)C(=O)OC1(c3ccc(O)cc3O2)c2ccc(O)cc2Oc2cc(O)ccc21</smiles>

图 29 探针 39 与生物硫醇类物质识别示意图

Figure 29 Schematic illustration of reaction of $\mathbf{3 9}$ with bio-thiols 


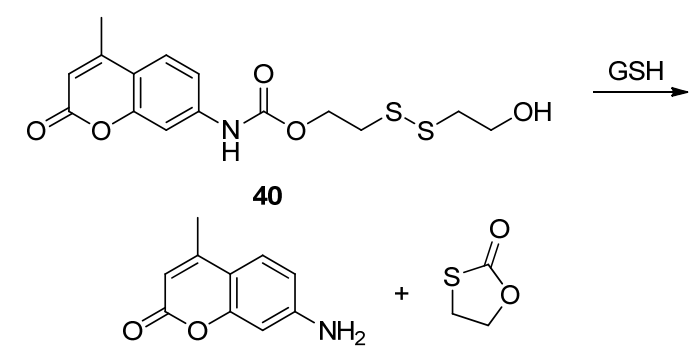

图 30 探针 40 与谷胱甘肽识别示意图

Figure 30 Schematic illustration of reaction of $\mathbf{4 0}$ with GSH

$40\left(20 \mu \mathrm{mol} \cdot \mathrm{L}^{-1}\right)$ 的 HEPES $\left(0.10 \mathrm{~mol} \cdot \mathrm{L}^{-1}, \mathrm{pH} 7.4\right)$ 缓冲溶 液中加入不同浓度的 GSH $\left(0 \sim 20 \mathrm{mmol} \cdot \mathrm{L}^{-1}\right)$, 苂光值会 随着 GSH 浓度的增加呈比例增加, 检测限为 $6 \mu \mathrm{mol}$ ・ $\mathrm{L}^{-1}$. 探针 40 具有定量检测细胞中 $\mathrm{GSH}$ 含量变化的潜 力.

2017 年, Shen 等 ${ }^{[56]}$ 报道了一种检测 GSH 的双光子 苂光探针 41 (图 31). 在探针 $\mathbf{4 1}$ 的溶液中加入 GSH 分别 在 350 和 $450 \mathrm{~nm}$ 处激发会分别在 460 和 $530 \mathrm{~nm}$ 处出现 两个苂光发射峰. 在探针 $41\left(5 \mu \mathrm{mol} \cdot \mathrm{L}^{-1}\right)$ 的 $\mathrm{PBS} / \mathrm{MeCN}$ $(V: V=1: 1, \mathrm{pH}$ 7.4) 溶液中加入不同浓度的 GSH $(0 \sim$ $\left.1000 \mu \mathrm{mol} \cdot \mathrm{L}^{-1}\right), 460$ 和 $530 \mathrm{~nm}$ 处的荧光发射峰均会随 着 GSH 的增加而增加, 检测限为 $2.9 \mu \mathrm{mol} \cdot \mathrm{L}^{-1}$. 此外, 探针 41 对 GSH 的检测具有高度的选择性, 并且探针 $\mathbf{4 1}$ 的设计思路也可以为双光子类荧光探针的设计研究提 供借鉴.

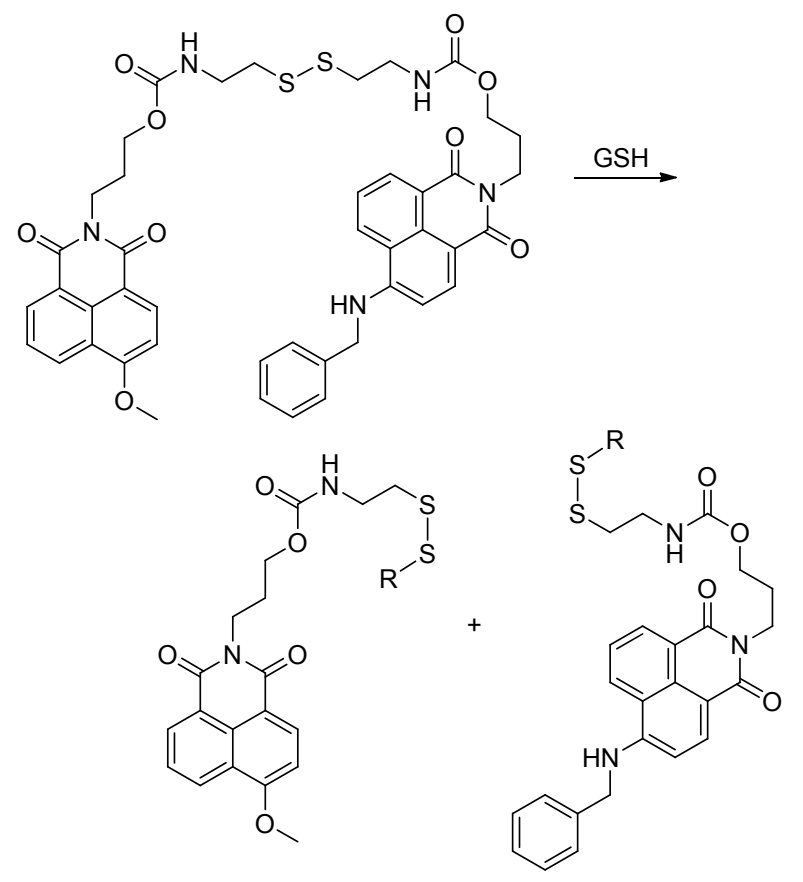

图 31 探针 41 与谷胱甘肽识别示意图

Figure 31 Schematic diagram of the response mechanism of $\mathbf{4 1}$ and GSH

2016 年, Song 等 ${ }^{[57]}$ 报道了基于卤素取代反应的检
测生物硫醇的苂光探针 42 (图 32). 该探针最大的优点 是，在不同的介质下区分结构较为相似的三种生物硫醇 (Cys、Hcy、GSH), 并且探针的水溶性较好. 探针 $\mathbf{4 2}$ 在 Tris- $\mathrm{HCl}\left(20 \mathrm{mmol} \cdot \mathrm{L}^{-1}, \mathrm{pH}\right.$ 7.4)缓冲溶液中加入等量的 Cys、Hcy、GSH 及其他 19 种氨基酸，结果表明在有 Cys 存在的情况下探针 $\mathbf{4 2}$ 的苂光值增加最大, 其次是 Hcy 和 GSH, 其他 19 种氨基酸与其基本无响应. 探针 $\mathbf{4 2}$ 通 过选择介质可以有效地检测并区分生物硫醇，具有较好 的应用前景.

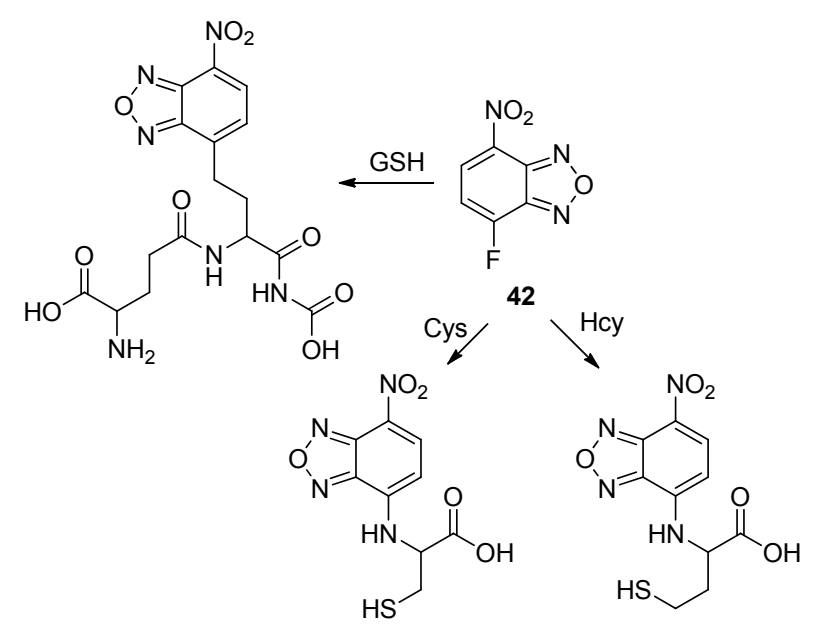

图 32 探针 42 与生物硫醇识别示意图

Figure 32 Schematic illustration of reaction of $\mathbf{4 2}$ with biothiols

2015 年, Dai 等 ${ }^{[58]}$ 报道了一种能区分检测 GSH 和 Cys、Hcy 的氯代香豆素醛菼光探针 43 (图 33). 通过与 探针 43 两个不同的位点反应，能有效地区分 GSH 和 Cys、Hcy. 在探针 $\mathbf{4 3}$ 的 PBS 缓冲溶液中分别加入 GSH、 Cys 和 Hcy, 溶液的颜色会发生比较明显的变化, 其中 加入 $\mathrm{GSH}$ 的溶液变为红色而加入 $\mathrm{Cys}$ 和 Hcy 的溶液则 变为浅黄色. 更重要的是, 探针 43 已经成功应用于活细 胞中生物硫醇的检测, 并且观察到不同生物硫醇所引起 的探针 43 颜色的变化.

2018 年, Hou 等 ${ }^{[59]}$ 报道了一种检测 GSH/Cys 的新型 的苂光探针 44 (图 34). 探针 44 对 GSH/Cys 的检测性能 明显高于包括 Hcy 在内的其他氨基酸, 此外探针 44 与 GSH/Cys 的响应时间均低于 $1 \mathrm{~h}$, 因此探针对 GSH/Cys 的检测具有较好的选择性和灵敏度. 在探针 44 (10 $\left.\mu \mathrm{mol} \cdot \mathrm{L}^{-1}\right)$ 的 $\mathrm{CH}_{3} \mathrm{CN} / \mathrm{H}_{2} \mathrm{O}(V: V=3: 7, \mathrm{PBS}$ 缓冲溶液, $10 \mathrm{mmol} \cdot \mathrm{L}^{-1}, \mathrm{pH}$ 7.4)溶液中加入不同浓度的 GSH/Cys $(0 \sim 3$ equiv. $)$, 探针 44 的荧光值随 GSH 或 Cys 浓度的变 化而变化, 检测限分别为 0.12 和 $0.13 \mu \mathrm{mol} \cdot \mathrm{L}^{-1}$. 此外, 探针 44 的细胞应用试验表明, 探针 44 具有较好的细胞 膜穿透性，能够应用于活细胞中 GSH/Cys 的检测. 


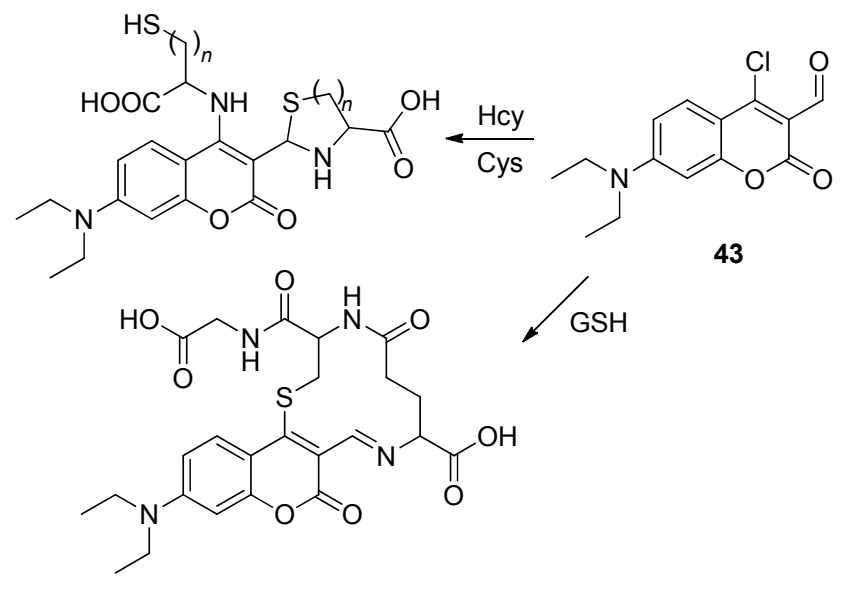

图 33 探针 43 与生物硫醇识别示意图

Figure 33 Schematic illustration of reaction of $\mathbf{4 3}$ with biothiols<smiles>[R]SSc1ccccn1</smiles><smiles>O=C1Cc2ccccc2S1</smiles>

图 34 探针 $\mathbf{4 4}$ 与 GSH/Cys 识别示意图

Figure 34 Schematic illustration of reaction of 44 with GSH/Cys

\section{5 其他识别机理}

1953 年, Du Vigneaud 等 ${ }^{[60]}$ 首次报道了运用自然化 学连接(NCL)法合成了生物活性多肽激素. 该方法主要 是应用两个多肽链在水溶液中反应形成硫酯键, 在自发 重排时, 硫酯键被转变成酰胺键. NCL 反应已被广泛应 用于许多领域, 如医药化学、生物学、生理学等. 2014 年, Lü 等 ${ }^{[61]}$ 报道了一种检测 Hcy 和 Cys 的基于 NCL 反 应的苂光探针 45(图 35). 在探针 45 的溶液中加入 Cys 后, 苂光的发射峰会由 $694 \mathrm{~nm}$ 蓝移至 $474 \mathrm{~nm}$ 处, 并伴 随着溶液由红色荧光变为莹绿色荧光. 探针 45 具有较 好的选择性和灵敏度, 其余 Hcy 和 Cys 的响应时间均在 $30 \mathrm{~min}$ 之内. 探针 45 对 Cys 和 Hcy 的检测限分别为 1.6 和 $1.8 \mathrm{nmol} \cdot \mathrm{L}^{-1}$. 此外, 探针 45 已经成功应用于活体 HepG2 细胞中 Cys 的检测.

自 1890 年Mond 首次报道金属配合物以来, 金属配 合物被广泛地应用于各个领域. 2016 年, $\mathrm{He}$ 等 ${ }^{[62]}$ 报道了 一种基于香豆素碳酰肼双核铜配合物的硫醇苂光探针

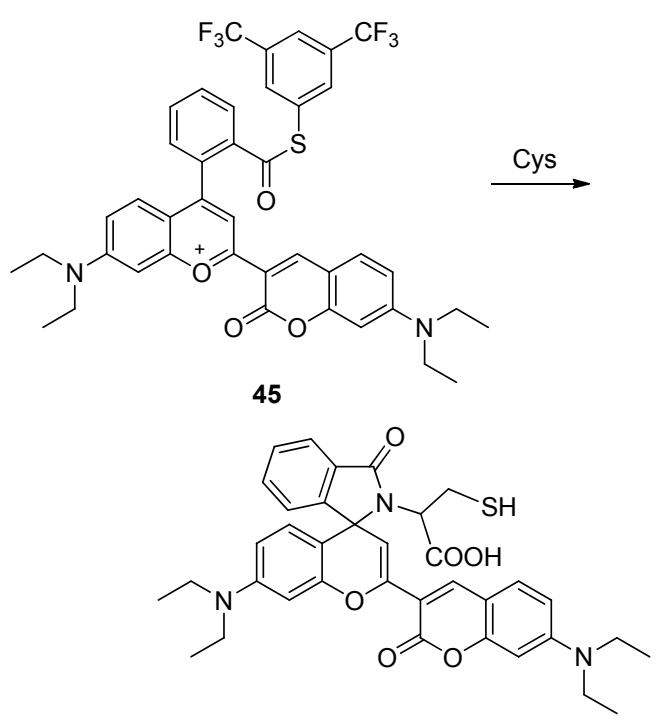

图 35 探针 45 与 Cys 识别示意图

Figure 35 Schematic illustration of reaction of $\mathbf{4 5}$ with Cys

46 (图 36). 铜离子的加入使得化合物与铜离子形成配合 物, 进而使得探针 46 的苂光被萃灭. 在探针 46 (10 $\left.\mu \mathrm{mol} \cdot \mathrm{L}^{-1}\right)$ 的 $\mathrm{CH}_{3} \mathrm{CN} / \mathrm{H}_{2} \mathrm{O}(V: V=3: 2) \mathrm{PBS}$ 缓冲溶液中 加入不同浓度的 GSH ( $0 \sim 2$ equiv. $)$, 探针 46 的苂光值会 随着 GSH 浓度的增加而增加. 探针 46 对生物硫醇类物 质具有较好的选择性，响应时间约为 $60 \mathrm{~min}$. 此外探针 46 成功地用于活体 $\mathrm{SiHa}$ 细胞的 GSH 显像.

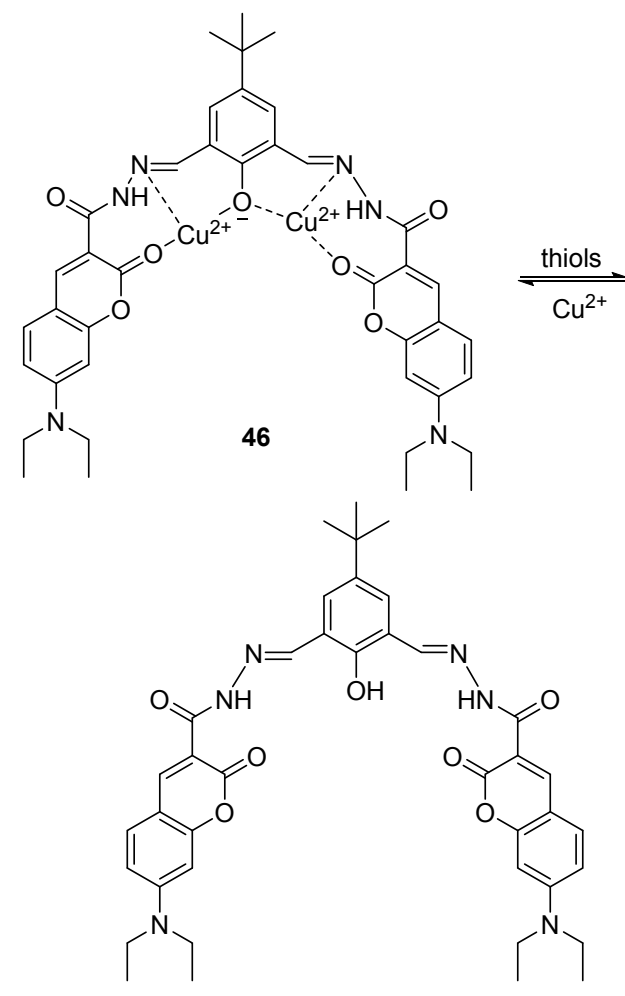

图 36 探针 46 与 GSH 识别示意图

Figure 36 Schematic illustration of reaction of $\mathbf{4 6}$ with GSH 
纳米材料作为一种新型材料, 近年来已成为研究的 热点, 并已应用于许多领域 ${ }^{[63,64]}$. 目前, 已经报道了各 种基于纳米粒子的荧光探针, 如量子点和金纳米团簇 $(\mathrm{AuCuS})$ (图 37) ${ }^{[65]} .2015$ 年, $\mathrm{Xu}$ 等 ${ }^{[66]}$ 报道了一种基于金 纳米团簇(AUNCS) 和聚合物保护金纳米粒子(AUNPS) 的苂光探针 47, 用于对 Cys 进行检测(图 37). AuNCS 具 有较强的荧光特性, 能通过福斯特共振能量转移 (FRET)抑制 AUNC 的苂光. Cys 可诱导聚 $N, N$-二甲基丙 烯酰胺金纳米颗粒(PDMAM AuNPS)聚集, 并恢复牛血 清白蛋白金纳米簇(BSA AuNCS)的一半荧光强度, 而其 它浓度较高的氨基酸难以提高其荧光强度. 探针 47 对 Cys 的检测限为 $3.6 \mu \mathrm{mol} \cdot \mathrm{L}^{-1}$, 此外探针 $\mathbf{4 7}$ 已经被成功 地应用于人体尿液中 Cys 的检测.

2018 年, Zhang 等 ${ }^{[67]}$ 报道了一种新型的检测硒代半 管氨酸的半花菁类苂光探针 48(图 38). 半花菁类分子的 席夫碱形式(SB)无苂光, 而质子化形式(PSB)都具有较 强的苂光信号, 根据这一特性利用硒醇调节水溶液中 $\mathrm{PSB} / \mathrm{SB}$ 平衡, 进而实现对光学信号的调控. 探针 $\mathbf{4 8}$ 对 硒代半胱氨酸的检测具有较高的选择性和灵敏度. 探针 48 对半胱氨酸的检测具有较高的选择性和灵敏度, 其 次探针 48 对细胞中内源性和外源性硒醇的检测都表现 出较好的成像效果.

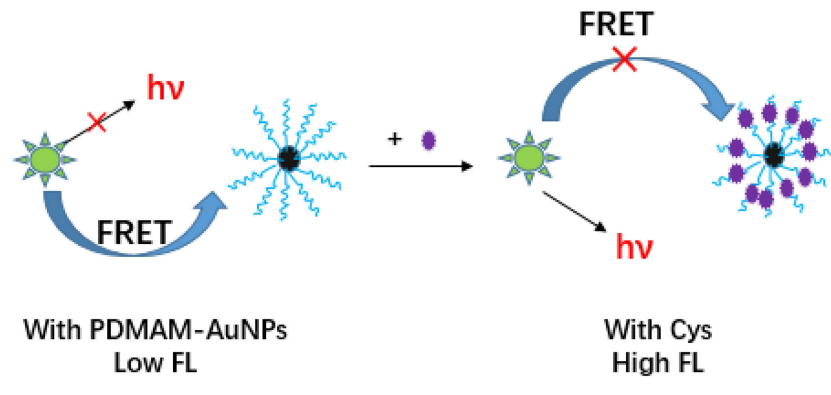

BSA-AuNCs

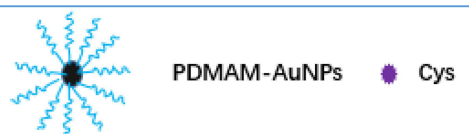

图 37 探针 $\mathbf{4 7}$ 与 $\mathrm{Cys}$ 识别示意图

Figure 37 Schematic illustration of reaction of $\mathbf{4 7}$ with Cys

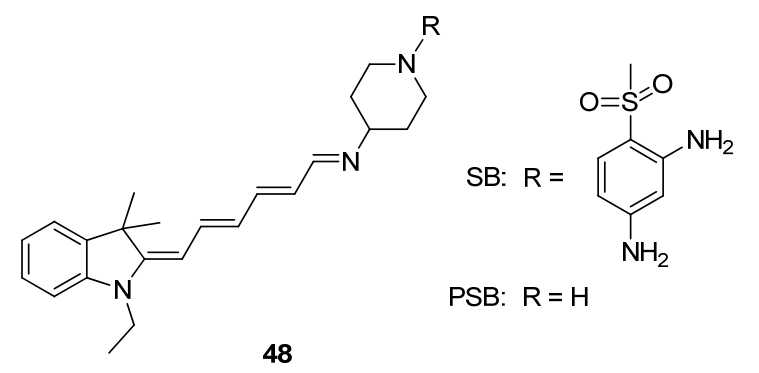

图 38 探针 $\mathbf{4 8}$ 结构示意图

Figure 37 Chemical structures of probe $\mathbf{4 8}$

\section{6 结语}

近年来, 統基荧光探针的研究进展非常迅速, 一些 较为新颖的方法不断地被报道. 然而, 对这些荧光探针 的研究仍然面临许多的挑战，如探针的水溶性问题、探 针的生理毒性、生物细胞自身苂光干扰性问题, 以及对 三种结构相似的氨基酸(半胱氨酸、HCY 和 GSH)区分检 测的问题等. 因此，预测未来的研究重点如下：(a)提高 探针的水溶性; (b)消除自动荧光在生物系统中的固有生 物分子中的干扰; (c)有效区分三种类似氨基酸; (d)发展 低生理毒性的体内光学成像的方法.

本文综述了近年来硫醇类苂光探针的研究进展，重 点讨论了颈基氨基酸 Cys、Hcy 和 GSH 的研究进展. 从 硫醇的迈克尔加成、醛的环化、裂解、取代、自然化学 连接、纳米材料等方面, 综述了一些具有代表性荧光探 针的传感机制、选择性、灵敏度、响应时间等.

\section{References}

[1] Schulz, J. B.; Lindenau, J.; Seyfried, J.; Dichgans, J. Eur. J. Biochem. 2000, 267(16), 4904.

[2] Haugaard, N. Ann. N. Y. Acad. Sci. 2000, 899(1), 148.

[3] Khoo, H. E.; Chen D. S.; Yuen, R. Toxicon 1998, 36(3), 469.

[4] Benathan, M.; Virador, V.; Furumura, M.; Kobayashi, N.; Panizzon, R. G.; Hearing, V. J. Cell. Mol. Biol. 1999, 45(7), 981.

[5] Zhang, Z. X.; Geng, D. Y.; Han, Q.; Liang, S. D.; Guo, H. R. J. Fish Biol. 2013, 83(5), 1287.

[6] Uchida, E.; Uemura, H.; Tanaka, T.; Nishikawa, S.; Uesugi, S.; Tanaka, A.; Morikawa, M.; Hayakawa, T.; Ikehara, M. Chem. Pharm. Bull. 1991, 39(1), 150.

[7] Kulwin, M. H. J. Invest. Dermatol. 1953, 20(3), 237.

[8] Sawamoto, O.; Kurisu, K.; Kuwamura, M.; Kotani, T.; Yamate, J.; Yamate, J. Exp. Toxicol. Pathol. 2003, 55(2 3), 121.

[9] Badawy, A. H.; Abdel.; Aal, S. F.; Samour, S. A. J. Egypt. Soc. Parasitol. 1989, 19(2), 563.

[10] Shahrokhian, S.; Lead Am. J. Anal. Chem. 2001, 73(24), 5972.

[11] Tan, B.; Venketasubramanian, N.; Vrooman, H.; Cheng, C. Y.; Wong, T. Y.; Ikram, M. K.; Chen, C.; Hilal, S. Alzheimera Dis. 2018, 62(2), 877.

[12] Doody, R. S.; Demirovic, J.; Ballantyne, C. M.; Chan, W.; Barber, R.; Powell, S.; Pavlik, V. Alzheimers Dement. (Amst.) 2015, 1(4), 464.

[13] Dietrichmuszalska, A.; Malinowska, J.; Olas, B.; Głowacki, R.; Bald, E.; Wachoeicz, B.; Rabe-Jabłońska, J. Neurochem. Res. 2012, 37(5), 1057.

[14] Elkhairy, L. Ueland, P. M.; Refsum, H.; Graham, I. M.; Vollset, S. E. Circulation 2001, 103(21), 2544.

[15] Stamler, J. S.; Slivka, A. Homocysteine Metabolism: From Basic Science to Clinical Medicine, Springer, US, 1997, pp. 211 222.

[16] Fraternale, A.; Casabianca, A.; Orlandi, C.; Cerasi, A.; Chiarantini, L.; Brandi, G.; Magnani, M. Antiviral Res.2002, 56(3), 263.

[17] Morgenstern, I.; Raijmakers, M. T. M.; Peters, W. H. M.; Hoensch, H.; Kirch,W. Dig. Dis. Sci. 2003, 48(10), 2083.

[18] Lv, Z.; Sun, Z.; Song, C.; Lu, S. M.; Chen,G.; You, G. M. Talanta 2016, 161, 228-237.

[19] Kawanishi, S. Biochim. Biophys. Acta, Gen. Subj. 2002, 1570(1), 47.

[20] Ercal, N.; Le, K.; Treeratphan, P.; Matthews, R. Biomed. Chromatogr. 2015, 10(1), 15.

[21] Baron, M.; Sochor, J. Int. J. Electrochem. Sci. 2013, 8(9), 11072.

[22] Sippel, T. O. Histochem. J. 1978, 10(5), 597. 
[23] Chen, J.; Jiang, X.; Carroll, S. L.; Huang, J.; Wang, J. Org. Lett. 2015, 17(24), 5978.

[24] Ren, W. X.; Han, J.; Pradhan, T.; Lim, J. Y.; Lee, J. H.; Lee, J.; Kim, J. H.; Kim, J. S. Biomaterials 2014, 35(13), 4157.

[25] Niu, L. Y.; Chen, Y. Z.; Zheng, H. R.; Wu, L. Z.; Tung, C. H.; Yang, Q. Z. Chem. Soc. Rev. 2015, 44(17), 6143.

[26] Fan, W.; Huang, X.; Shi, X.; Wang, Z.; Lu, Z. L.; Fan, C. H.; Bo, Q. B. Spectrochim. Acta, Part A 2017, 173, 918.

[27] Dai, X.; Zhang, T.; Liu, Y. Z.; Yan, T.; Li, Y.; Miao, J. Y.; Zhao, B. X. Sens. Actuators, B 2015, 207, 872.

[28] Lim, S. Y.; Yoon, D. H.; Ha, D. Y.; Ahn, J. M.; Kim, D.; Kown, H.; Ha, H. J.; Kim, H. J. Sens. Actuators, B 2013, 188(11), 111.

[29] Huo, F.; Kang, J.; Yin, C.; Yue, Y. K.; Hao, J. S.; Chao, J. B.; Liu, D. S. Sens. Actuators, B 2015, 207(7), 139.

[30] Qu, L.; Yin, C.; Huo, F.; Li, J.; Chao, J.; Zhang, Y. Sens. Actuators, B 2014, 195(5), 246

[31] Shen, Y.; Liu, C.; Zhang, Y.; Zhang, X.; Zhang, C.; Jin, J.; Liu, X.; Li, H.; Yao, S. J. Anal. Methods Chem. 2015, 7(15), 6419.

[32] Liu, T.; Huo, F.; Yin, C.; Li, J.; Chao, J.; Zhang, Y. Dyes Pigm. 2016, 128, 209.

[33] Zhao, Y.; Xue, Y.; Li, H.; Zhu, R.; Ren, Y.; Shi, Q.; Wang, S.; Guo, W. Spectrochim. Acta, Part A 2017, 175, 215.

[34] Yue, Y.; Huo, F.; Peng, N.; Zhang, Y.; Chao, J.; Meng, X.; Yin, C. J. Am. Chem. Soc. 2017, 139(8), 3181.

[35] Sok, N.; Nikolantonaki, M.; Guyot, S.; Nguyen, T. D.; Viaux, A. S.; Bagala, F.; Rousselin, Y.; Husson, F.; Gougeon, R.; Saurel, R. Sens. Actuators, B 2016, 242, 865.

[36] Long, L.; Zhou, L.; Wang, L.; Meng, S.; Gong, A.; Du, F.; Zhang, C. Org. Biomol. Chem. 2013, 11(47), 8214.

[37] Dai, C. G.; Du, X. J.; Song, Q. H. J. Org. Chem. 2015, 80(24), 12088.

[38] Ou-Yang, J.; Li, C. Y.; Li, Y. F.; Fei, J.; Xu, F.; Li, S. J.; Nie, S. X. Sens. Actuators, B 2017, 240, 1165.

[39] Meng, X.; Ye, W.; Wang, S.; Feng,Y; Chen, M.; Zhu, M.; Guo, Q. Sens. Actuators, B 2014, 201(4), 520.

[40] Chen, C.; Liu, W.; Xu, C.; Liu, W. Biosens. Bioelectron. 2016, 85, 46.

[41] Zhang, J.; Lv, Y.; Zhang, W.; Ding, H.; Liu, R.; Zhao, Y.; Zhang, G.; Tian, Z. Talanta 2016, 146, 41.

[42] Kong, F.; Liu, R.; Chu, R.; Wang, X.; Xu, K.; Tang, B. Chem. Commun. 2013, 49(80), 9176.

[43] Gong, D.; Tian, Y.; Yang, C.; Iqbal, A.; Wang, Z.; Liu, W.; Qin, W.;
Zhu, X.; Guo, H. Biosens. Bioelectron. 2016, 85, 178.

[44] Gao, H.; Li, Z.; Zhao, Y.; Qi, H.; Zhang, C. Sens. Actuators, B 2017, 245, 853.

[45] Kim, S. K.; Gupta, M.; Lee, H. I. Sens. Actuators, B 2017, 257, 728.

[46] Wang, J.; Cheng, Z.; Zhang, J.; Zhu, X.; Liu, X.; Wang, Q.; Zhang, H. Spectrochim. Acta, Part A 2016, 166, 31

[47] Chen, S.; Li, H.; Hou, P. Tetrahedron 2017, 73(5), 589.

[48] Wei, M.; Yin, P.; Shen, Y.; Zhang, L.; Deng, J.; Xue, S.; Li, H.; Guo, B.; Zhang, Y.; Yao, S. Chem. Commun. 2013, 49(41), 4640.

[49] Liu, Y.; Xiang, K.; Guo, M; Tian, B.; Zhang, J. L. Tetrahedron Lett. 2016, 57(23), 2478.

[50] Fan, J.; Han, Z.; Yao, K.; Peng, X. J. Sci. Rep. 2016, 6, 19562.

[51] Yoshida, M.; Kamiya, M.; Yamasoba, T.; Urano, Y. Bioorg. Med. Chem. Lett. 2014, 24(18), 4363.

[52] Wang, K.; Leng, T.; Liu, Y.; Wang, C.; Shi, P.; Shen, Y.; Zhu, W. H. Sens. Actuators, B 2017, 248, 338.

[53] Xu, K.; Qiang, M.; Gao, W.; Su, R. Chem. Sci. 2013, 4(3), 1079.

[54] Zheng, L. Q.; Li, Y.; Yu, X. D.; Xu, J. J.; Chen, H. Y. Anal. Chim. Acta 2014, 850, 71.

[55] Lim, S. Y.; Na, M. J.; Kim, H. J. Sens. Actuators, B 2013, 185(8), 720 .

[56] Shen, W.; Ge, J.; He, S.; Zhang, R.; Zhao, C.; Fan, Y.; Yu, S.; Liu, B.; Zhu, Q. Chem. Asian J. 2017, 12(13), 1532.

[57] Song, L.; Ma, L. M.; Qian, S.; Zhang, W. B.; Lan, M. B.; Qian, J. H. Chin. Chem. Lett. 2016, 27(3), 330.

[58] Dai, X.; Wang, Z. Y.; Du, Z. F.; Cui, J.; Miao, J. Y.; Zhao, B. X. Anal. Chim. Acta 2015, 900, 103.

[59] Hou, X.; Li, Z.; Li, B.; Liu, C.; Xu, Z. Sens. Actuators, B 2018 , 260,295 ..

[60] Vigneaud, V. D.; Ressler, C.; Swan, C. J. M. J. Am. Chem. Soc. 1953, 75(19), 4879.

[61] Lv, H.; Yang, X. F.; Zhong, Y.; Guo, Y.; Li, Z.; Li, H. Anal. Chem. 2014, 86(3), 1800

[62] He, G.; Li, J.; Yang, L.; Hou, C.; Ni, T.; Yang, Z.; Qian, X.; Li, C. Plos One. 2016, 11(2), e0148026.

[63] Sun, Q.; Sheng, J. Chem. Ind. Eng. Prog. 1997, 10(1), 48.

[64] Zhang, L.; Wang, E. Nano Today 2014, 9(1), 132.

[65] T Tian, D.; Qian, Z.; Xia, Y.; Zhu, C. Langmuir 2012, 28(8), 3945.

[66] Xu, X.; Qiao, J.; Li, N.; Qi, L.; Zhang, S. Anal. Chim. Acta 2015, $879,97$.

[67] Zhang, S.; Qin, W.; Liu, X.; Zhang, J. mBio 2017, 8(6), e01620.

(Li, L.; Fan, Y.) 